\title{
Joint-Detection and Interference Cancellation Based Burst-by-Burst Adaptive CDMA Schemes
}

\author{
Ee-Lin Kuan, Soon Xin Ng, and Lajos Hanzo, Senior Member, IEEE
}

\begin{abstract}
Spread adaptive quadrature amplitude modulated (AQAM) code-division multiple access (CDMA) is proposed as a powerful means of exploiting the time-variant channel capacity fluctuations of wireless channels. It is studied in comparison to variable spreading factor (VSF)-based techniques. These adaptive-rate transmission methods are compared in the context of joint detection and interference cancellation assisted adaptive CDMA (ACDMA) systems. More explicitly, we exploit the time-variant channel quality of mobile channels by switching either the modulation mode (AQAM) or the spreading factor (VSF) on a burst-by-burst basis. The most appropriate modulation mode or spreading factor is chosen based on the instantaneous channel quality estimated. The chosen modem mode or spreading factor is communicated to the remote communicator either through explicit signalling or extracted at the receiver using blind detection techniques. The multiuser joint detector (JD) and the successive interference cancellation (SIC) receiver are compared in the context of these adaptive schemes, with the conclusion that the JD outperformed the SIC receiver in the ACDMA schemes at the cost of increased complexity. Finally, the performance of the uncoded AQAM JD-CDMA scheme is also compared to that of adaptive trellis coded modulation (TCM) assisted AQAM JD-CDMA, which allows us to incorporate adaptive channel coding without any bandwidth expansion. We also show that in the particular scenario studied, adaptive TCM outperformed adaptive turbo TCM since the system was designed for maintaining a low turbo-interleaver delay.
\end{abstract}

Index Terms-Burst-by-burst adaptive code-division multiple access (CDMA), joint detection CDMA, parallel interference cancellation (PIC), successive interference cancellation (SIC), successive and parallel interference cancellation.

\section{INTRODUCTION}

$\mathbf{M}$ OBILE radio signals are subject to propagation path loss as well as slow fading and fast fading. Due to the nature of the fading channel, transmission errors occur in bursts when the channel exhibits deep fades. In mobile communications systems, power-control techniques are used to mitigate the effects of path loss and slow fading. However, in order to counter the problem of fast fading and cochannel interference, agile powercontrol algorithms are required [1]. Another technique that can be used to overcome the problems due to fading is adaptive-rate transmission [2]-[6], where the information rate is varied according to the quality of the channel. When the channel quality is low, a lower information rate is chosen in order to reduce the number of errors. Conversely, when the channel quality is high,

Manuscript received January 22, 2001; revised May 15, 2002. This work was supported by the European Union and by EPSRC, Swindon, U.K.

The authors are with the Department of Electronics and Computer Science, University of Southampton, S017 1BJ Southampton, U.K. (e-mail: lh@ecs. soton.ac.uk).

Digital Object Identifier 10.1109/TVT.2002.804842 a higher information rate is used to increase the throughput of the system. This method is similar to multirate transmission, except that in this case, the transmission rate is modified according to the channel quality instead of the service required by the mobile user. Different methods of multirate transmission have been proposed by Ottosson and Svensson [7]. According to the multicode method, multiple codes are assigned to a user requiring a higher bit rate [7]. Multiple data rates can also be provided by a multiple processing-gain scheme, where the chip rate is kept constant but the data rates are varied by changing the processing gain of the spreading codes assigned to the users. Performance comparisons for both of these schemes have been carried out by Ottosson et al. [7] and Ramakrishna et al. [8], demonstrating that both schemes achieved similar performance. Saquib et al. [9] and Johansson et al. [10] have also investigated the employment of the decorrelating detector and the successive interference cancellation receiver for multirate code-division multiple access (CDMA) systems.

Adaptive-rate transmission schemes, where the transmission rate is adapted according to the channel quality, have also been proposed. Abeta et al. [11] conducted investigations into an adaptive packet transmission based CDMA scheme, where the transmission rate was modified by varying the channel code rate and the processing gain of the CDMA user, employing the carrier-to-(interference plus noise) ratio (CINR) as the switching metric. When the channel quality was favorable, the instantaneous transmission bit rate was increased; and conversely, the instantaneous bit rate was reduced when the channel quality dropped. To maintain a constant overall bit rate, when a high instantaneous bit rate was employed, the duration of the transmission burst was reduced. Conversely, when the instantaneous transmission bit rate was low, the duration of the burst was lengthened. This resulted in a decrease in interference power, which translated to an increase in system capacity. Hashimoto et al. [12] extended this work to show that the proposed system was capable of achieving a higher capacity with a smaller handoff margin and lower average transmitter powers. In these schemes, the conventional RAKE receiver was used for the detection of the data symbols. Kim [6] analyzed the performance of two different methods of combating the mobile channel's variations, which were the adaptation of the transmitter power to compensate for channel variations or the switching of the information rate to suit the channel conditions. Using a RAKE receiver, it was demonstrated that rate adaptation provided a higher average information rate than power adaptation for a given average transmit power and a given bit error rate (BER). A range of other design issues of adaptive CDMA can be found, for example, in [13]-[17]. 
In this paper, we also propose to vary the information rate in accordance with the channel quality. However, in comparison to conventional power control techniques-which may disadvantage other users by inflicting an increased interference due to the associated higher transmit power in an effort to maintain the quality of the reference link considered-the proposed technique does not disadvantage other users and yet increases the network capacity [18]. The instantaneous channel quality can be estimated at the receiver, and the chosen information rate can then be communicated to the transmitter via explicit signalling in a closed-loop scheme. Conversely, in an open-loop scheme, by assuming reciprocity in the uplink and downlink channels of wide-band time-division duplex (TDD) CDMA systems, the information rate for the downlink transmission is chosen according to the channel quality estimate related to the uplink and vice versa. Channel reciprocity issues in TDD/CDMA systems have been investigated by Miya et al. [19], Kato et al. [20], and Jeong et al. [21]. In our investigations, we have employed the FRAMES Mode 1 time-division multiple-access (TDMA)/CDMA [22] system, which is a frequency-division duplexing mode. However, we have assumed perfect channel impulse response (CIR) estimation, and as such, our investigations are also valid for TDD/CDMA systems where perfect CIR estimation is also assumed.

The proposed adaptive quadrature amplitude modulated (AQAM)-based joint detector (JD)-CDMA system is compared to variable spreading factor (VSF)-based transmissions. AQAM is an adaptive-rate technique, whereby the data modulation mode is chosen according to some criterion related to the channel quality. On the other hand, in VSF transmission, the information rate is varied by adapting the spreading factor of the CDMA codes used while keeping the chip rate constant. Further elaborations on these two methods will be given in subsequent sections. The multiuser receivers used for data detection are JD receivers and successive interference cancellation (SIC) receivers. The performance of these two receivers with regards to the adaptive-rate schemes will be explored.

The design issues of modulation mode synchronization between the transmitter and the receiver were discussed, for example, in the excellent contributions of Lau et al. [15], [16], [23]. Explicitly, in [15], the modulation mode to be used by the transmitter was determined on a symbol-by-symbol basis by the channel state predictor (CSP). By using two identical CSPs at the transmitter and the receiver, the same modulation mode sequence can be generated by feeding the two CSPs with the delay-compensated stream of previous channel states for predicting the next channel state. Hence, the mode synchronization between the transmitter and the receiver was maintained in [15] by synchronizing the feedback delay. On the other hand, a closed-loop control scheme was used for mantaining mode synchronization between the transmitter and the receiver in [23]. More specifically, a control word signalling the requested transmission mode for each of the symbols in a transmission burst was embedded into the burst header, where the control words belonging to several bursts were protected by Bose-Chaudhuri-Hocquenheim codes, interleaved, and quaternary phase-shift keying modulated. As a design alternative, Otsuki et al. [24] proposed using a four-chip
Walsh code for signalling the modem modes, where the codes were detected with the aid of maximum likelihood detection. They suggested transmitting the Walsh codes using binary phase-shift keying (BPSK) modulation in order to maintain the highest possible integrity of the mode signalling information. In a further contribution, Torrance et al. [25] advocated the employment of a specific uneven error protection based PSK modulation constellation having five phasors for signalling a set of five AQAM modes, which were nonuniformly distributed on the 5-PSK constellation ring. This scheme had the advantage that the phasor points signalling the low-throughput AQAM modes under hostile channel conditions were spaced far apart for the sake of minimizing their corruption probability. By contrast, the phasor points signalling the high-throughput AQAM modes under benign channel conditions were more densely spaced, since these were typically activated under conditions of high signal-to-noise ratio (SNR), which guaranteed a low probability of corruption. Given the above range of AQAM mode signalling solutions in the literature, in this contribution, we refrained from considering this issue and have assumed perfect modulation mode signalling.

The remainder of this paper is organized as follows. Section II describes the proposed joint detection assisted AQAM and VSF adaptive CDMA (ACDMA) systems, while Section III provides an overview of interference cancellation assisted receivers. In Section IV, trellis coded modulation (TCM) and turbo TCM (TTCM) are invoked, and the higher performance TCM scheme is utilized for assisting the AQAM JD-CDMA system in order to obtain an additional throughput gain without any bandwidth expansion. Our discussions and concluding remarks are summarized in Section V.

\section{JOINT DETECTION}

Joint detection receivers [26]-[30] constitute a class of multiuser receivers that were developed based on conventional equalization techniques [31] used for mitigating the effects of intersymbol interference (ISI). These receivers utilize the CIR estimates and the knowledge of the spreading sequences of all the users in order to reduce the level of multiple-access interference (MAI) in the processed signal.

By concatenating the data symbols of each CDMA user successively, as though they were transmitted by one user, we can apply the principles of conventional TDMA-oriented channel equalization [31] to multiuser detection. For $K$ users transmitting $N$ symbols each, the concatenated data vector is

$$
\mathbf{d}=d_{1}^{1}, d_{2}^{1}, \ldots, d_{N}^{1} ; d_{1}^{2}, d_{2}^{2}, \ldots, d_{N}^{2} ; \ldots, d_{1}^{K}, \ldots, d_{N}^{K}
$$

where $\mathbf{d}$ represents the data frame and $d_{n}^{k}$ represents the $n$th symbol of the $k$ th user for $n=1,2, \ldots, N$ and $k=1,2, \ldots, K$.

The received signal $\mathbf{y}$ after down-conversion may be written in matrix form as follows:

$$
\mathbf{y}=\mathbf{A d}+\mathbf{n}
$$

where $\mathbf{y}$ is a column vector and $\mathbf{n}$ represents the noise vector, consisting of noise samples corrupting the received 
signal. The column vectors $\mathbf{a}_{j}$ of the system matrix $\mathbf{A}$, where $j=1,2, \ldots, j, \ldots, K N$, are given by

$$
\mathbf{a}_{j}=\left(\begin{array}{c}
\mathbf{0}_{Q \cdot(n-1)} \\
\mathbf{c}^{(k)} * \mathbf{h}^{(k)} \\
\mathbf{0}_{Q \cdot(N-n)}
\end{array}\right)
$$

where $j=(k-1) N+n$, for $k=1,2, \ldots, K$ and $n=$ $1,2, \ldots, N$. Furthermore, the vector $\left(\mathbf{c}^{(k)} * \mathbf{h}^{(k)}\right)$ represents the combined impulse response for the $k$ th user given by the discrete-time linear convolution of $\mathbf{h}^{(k)}$-the chip-rate-based CIR estimates of the $k$ th user-with the spreading sequence $\mathbf{c}^{(k)}$ of the same user. Finally, the notations $\mathbf{0}_{Q \cdot(n-1)}$ and $\mathbf{0}_{Q \cdot(N-n)}$ indicate all-zero column vectors of length $Q \cdot(n-1)$ and $Q \cdot(N-$ $n$ ), respectively. To simplify our discussions, it is assumed that for a given user, the same spreading sequence is used for all the data symbols in the same transmission burst and that the CIR varies slowly, such that the CIR estimates can be considered time-invariant throughout one data burst. The column vector $\mathbf{n}$ represents the noise samples corrupting the down-sampled signal.

We have considered four basic joint detection algorithms in our investigations [27], and we have concluded that minimum mean square error block decision feedback equalization (MMSE-BDFE) provided the best performance for the COST 207 [32] Bad Urban channel model with perfect CIR estimation. Additionally, the detection complexity was similar for all four algorithms. Therefore, in our further investigations, we have used the MMSE-BDFE. In other cases, the linear joint detection schemes may show better performance, especially at low $E_{s} / N_{0}$ values, where there is a higher probability of error propagation in the feedback scheme. However, these linear JD receivers are also amenable to the ACDMA schemes that will be discussed subsequently. Upon exploiting the so-called orthogonality principle [33], it can be shown that the linear MMSE joint detector obtains the data estimates $\hat{\mathbf{d}}$ according to

$$
\hat{\mathbf{d}}=\left(\mathbf{A}^{H} \mathbf{R}_{n}^{-1} \mathbf{A}+\mathbf{R}_{d}^{-1}\right)^{-1} \mathbf{A}^{H} \mathbf{R}_{n}^{-1} \mathbf{y}
$$

where $\mathbf{R}_{n}=\mathbf{E}\left[\mathbf{n n}^{H}\right]$ is the noise covariance matrix and $\mathbf{R}_{d}=$ $\mathbf{E}\left[\mathbf{d} \mathbf{d}^{H}\right]$ is the data covariance matrix. In the MMSE-BDFE algorithm, Cholesky decomposition [34] is performed on the matrix of $\left(\mathbf{A}^{H} \mathbf{R}_{n}^{-1} \mathbf{A}+\mathbf{R}_{d}^{-1}\right)$, resulting in a lower triangular and an upper triangular matrix of $\mathbf{L}$ and $\mathbf{U}$ respectively, where

$$
\mathbf{A}^{H} \mathbf{R}_{n}^{-1} \mathbf{A}+\mathbf{R}_{d}^{-1}=\mathbf{L} \mathbf{U} .
$$

Substituting (5) into (4), we obtain

$$
\hat{\mathbf{d}}=\mathbf{U}^{-1} \mathbf{L}^{-1} \mathbf{A}^{H} \mathbf{R}_{n}^{-1} \mathbf{y}
$$

where the received vector $\mathbf{y}$ is first processed by a whitening matched filter matrix given by $\left(\mathbf{A}^{H} \mathbf{R}_{n}^{-1}\right)$. Next, the resultant vector is multiplied with the feed-forward matrix of $\mathbf{L}^{-1}$ in order to remove some of the interference in the signal. The data estimates are then fed back into the detector using the elements in the matrix $\mathbf{U}$ as the feedback filter tap coefficients. The feedback process can be expressed as

$$
d_{i}^{\prime}=\frac{\hat{y}_{i} \sum_{j=i+1}^{J=K N} U_{i, j} \hat{d}_{j}}{U_{i, i}}
$$

where $d_{i}^{\prime}$ is the $i$ th data symbol estimate of the data vector $\mathbf{d}, \hat{d}_{i}$ is the $i$ th data symbol that has been quantized by the demodulator, $\hat{y}_{i}$ is the $i$ th element of the received vector $\hat{\mathbf{y}}$ at the output of the feed-forward filter, and $U_{i, j}$ is the element in the $i$ th row and $j$ th column of the matrix $\mathbf{U}$.

\section{A. JD-Based Adaptive Code-Division Multiple Access}

In QAM [31], $n$ bits are grouped to form a signalling symbol and $m=2^{n}$ different symbols fully convey all combinations of the $n$ bits. These $m$ symbols are arranged in a constellation to form the $m$-QAM scheme. The square QAM modes of BPSK (2-QAM), 4-QAM, and 16-QAM convey 1, 2, and 4 bits per symbol, respectively. However, for a given channel signal-tonoise ratio (SNR), the bit error rate (BER) performance degrades as the number of constellation points increases, since the average distance between adjacent constellation points is reduced, which increases the probability of demodulation errors.

Burst-by-burst AQAM [31] is a technique that attempts to increase the average throughput of the system by switching between modulation modes depending on the state of the channel. When the channel quality is favorable, a modulation mode having a high number of constellation points is used to transmit as many bits per symbol as possible in order to increase the throughput. Conversely, when the channel is hostile, the modulation mode is switched to one using a small number of constellation points in order to reduce the error probability and to maintain a target BER.

Previous research in AQAM schemes for TDMA transmissions has been carried out by Webb and Steele [5], Sampei et al. [35], Goldsmith and Chua [36], and Torrance et al. [37]. This work has been extended to wideband channels, where the received signal also suffers from ISI in addition to amplitude and phase distortion due to the fading channel. Wong and Hanzo [38] proposed a joint adaptive modulation and equalization scheme, where a channel equalizer was used for mitigating the effects of ISI imposed on the signal based on a CIR estimate.

Here we propose to combine joint detection CDMA [27] with AQAM by modifying the approach used by Wong et al. [38]. Joint detection is particularly suitable for combining with AQAM because the implementation of the joint detection algorithms does not require any knowledge of the modulation mode used. The joint detection algorithm utilizes only the CIR estimates and the spreading sequences of all the users. Therefore, the joint detection receivers are suitable for combining with AQAM, since they do not have to be reconfigured each time the modulation mode is switched and the complexity is independent of the modulation mode used.

In our work, simulation tools were used for assessing the performance of our proposed schemes. The simulation parameters used are listed in Table I, and a spreading factor of length $Q=16$ is used in this section. The spreading sequences were generated pseudorandomly for each of the users. In our investigations, the COST 207 [32] channel models developed for the Global System of Mobile Communications (GSM) were employed. Although due to the orthogonal spreading sequences and to the resultant high chip rate the number of resolvable multipath components is higher in our CDMA system than in GSM, nonetheless we used the COST 207 models, since these are 

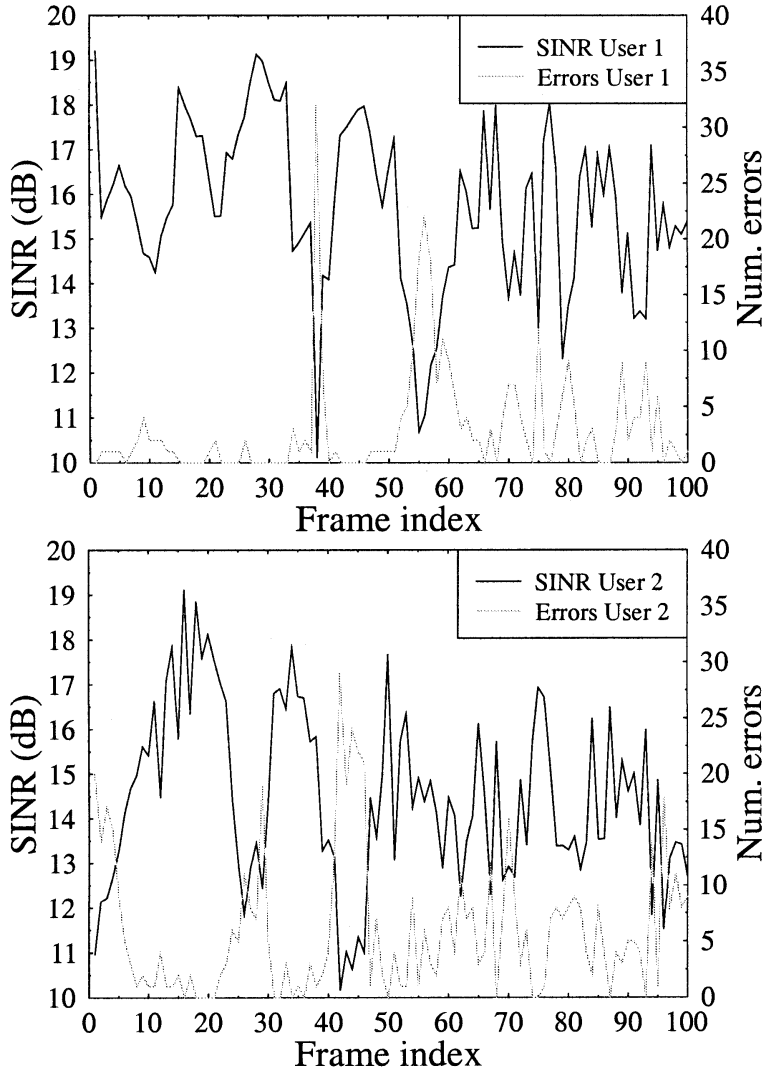

Fig. 1. Comparison of the SINR at the output of the MMSE-BDFE for different users versus transmission frame index, i.e., time. The number of errors corresponding to the SINR is also shown. There were two users in the system, transmitting at $E_{s} / N_{0}=10 \mathrm{~dB}$, and the 4-QAM modulation mode was used. The remaining system parameters are summarized in Table I, and the CIR is plotted in Fig. 2.

widely used in the community. A seven-path Bad Urban COST 207 channel was used, and the CIR can be seen in Fig. 2. Perfect channel estimation was assumed, providing an upper bound performance estimate. The uplink scenario was investigated by allowing each user to have independent fading in its channel.

Fig. 1 shows the variation in signal-to-(interference plus noise) ratio (SINR) at the output of the MMSE-BDFE and the number of errors per burst for different users over a period of time, where $E_{s} / N_{0}=10 \mathrm{~dB}$ and the 4-QAM modulation mode was used. The remaining system parameters are summarized in Table I, while the CIR profile is plotted in Fig. 2. Fig. 1 shows that the output SINR of the MMSE-BDFE varies over time and that the variation in output SINR is different for different users. This suggests potential advantages in combining AQAM with JD-CDMA.

To choose the modulation mode for AQAM transmission, the SINR at the output of the MMSE-BDFE was estimated by modifying the expression given in [27] with the assumptions that the data bits and noise values were uncorrelated, i.e., $\mathbf{R}_{d}=\mathbf{D}_{g}$ and $\mathbf{R}_{n}=\sigma^{2} \mathbf{I}$. For the $j$ th symbol in the data vector, $\mathbf{d}$ of (1), its signal power is represented by $g_{j}^{2}$. Therefore, the data covariance matrix $\mathbf{R}_{d}$ is a diagonal matrix $D_{g}$, where each element on its diagonal is the signal power, i.e., $\left[\mathbf{D}_{g}\right]_{j, j}=g_{j}^{2}$. The symbol $\sigma^{2}$ represents the noise variance, and the notation $\mathbf{I}$ indicates the
TABLE I

SimUlation PARAMETERS FOR THE ADAPTIVE CDMA SYSTEMS. THE CIR PROFILE Is SHOWN IN FIG. 2

\begin{tabular}{l|l}
\hline Parameter & Value \\
\hline \hline Channel type & COST 207 Bad Urban [32] \\
\hline Paths in channel & 7 \\
\hline Doppler frequency & $80 \mathrm{~Hz}$ \\
\hline Spreading sequence & Pseudo-random \\
\hline Chip rate & 2.167 MBaud \\
\hline Frame burst & FMA 1 Spread burst 1 [22] \\
\hline Burst duration & $577 \mu \mathrm{s}$ \\
\hline
\end{tabular}

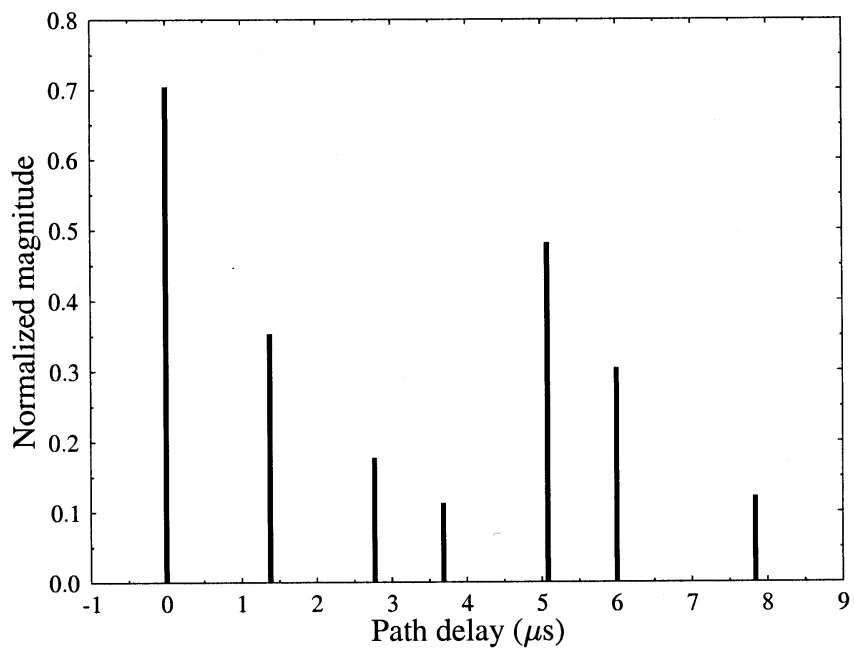

Fig. 2. Bad Urban seven-path COST 207 channel used in the simulations [32].

identity matrix. Then the output SINR for the $n$th symbol of the $k$ th user is given by

$$
\gamma_{o}(k, n)=g_{j}^{2}[\mathbf{D}]_{j, j}^{2}-1
$$

for $j=n+N(k-1)$. The square matrix $\mathbf{D}$ is a diagonal matrix, where all the elements on the main diagonal are the same real-valued elements as those on the main diagonal of the matrix $\mathbf{U}$ in (5). The notation $[\mathbf{D}]_{j, j}$ indicates the element in the $j$ th row and $j$ th column of the matrix $\mathbf{D}$. The average output SINR of an entire burst for the $k$ th user is obtained by summing and averaging the SINR values for each symbol

$$
\gamma_{o}(k)=\frac{1}{N} \sum_{n=1}^{N} \gamma_{o, \mathrm{JD}}(k, n)
$$

where $N$ represents the number of data symbols per user per burst.

We investigated triple-mode AQAM schemes. The modulation mode was switched among BPSK, 4-QAM, and 16-QAM, allowing the bit rate to vary from the minimum of 1 bit per symbol to the maximum of 4 bits per symbol. The output SINR was estimated on a burst-by-burst basis and used as the 
switching criterion. The rules used to switch between the three modes were as follows:

$$
\text { Mode }= \begin{cases}\text { BPSK, } & \text { for } \gamma_{o}(k)<t_{1} \\ \text { 4-QAM, } & \text { for } t_{1} \leqslant \gamma_{o}(k)<t_{2} \\ 16-\mathrm{QAM}, & t_{2} \leqslant \gamma_{o}(k)\end{cases}
$$

where $t_{1}$ and $t_{2}$ represented the switching thresholds, which were set according to the target BER requirements. In 1996, Torrance et al. [39] proposed a set of mode-switching levels s optimized for achieving the highest average bits per symbol (BPS) throughput while maintaining the target average BER. Their method was based on defining a cost function for transmission over narrow-band Rayleigh channels, which incorporated both the PBS throughput and the target average BER of the system. Powell's optimization was invoked for finding a set of switching thresholds, which were constant, regardless of the actual channel SNR encountered, i.e., from the prevalent channel conditions.

However, in 2001, Choi et al. [40] recognized that a higher BPS throughput can be achieved if, under high channel SNR conditions, the activation of high-throughput AQAM modes is further encouraged by lowering the switching thresholds. More explicitly, a set of SNR-dependent mode-switching levels was proposed [40], which keeps the average BER of AQAM constant while maximizing the achievable throughput. However, the set of switching levels derived in [39] and [41] is based on Powell's multidimensional optimization technique [42], and hence the optimization process may become trapped in a local minimum. This problem was overcome by Choi et al. upon deriving an optimum set of switching levels [40] when employing the Lagrangian multiplier technique. It was shown that this set of switching levels results in the global optimum in the sense that the corresponding AQAM scheme obtains the maximum possible average BPS throughput while maintaining the target average BER. A further approach was proposed by Lau in [43]. However, since the optimum switching thresholds depend on the shape of the channel impulse response, in this contribution the thresholds were set experimentally by finding the SNR values, where the constituent fixed-mode QAM schemes were capable of maintaining the required target BER.

In [15], Lau and Maric proposed an eight-mode adaptive modulation scheme for direct-sequence CDMA using M-ary orthogonal modulation. More specifically, a rate-1/9 code and a $2^{9}$-ary orthogonal modulator were employed in Mode 0 , while a rate- $1 / 2$ code and a $2^{2}$-ary orthogonal modulator were employed in Mode 7 . The adaptive mode-switching thresholds in this scheme were chosen in 3-dB steps. The convenience of this was that due to the fixed relationship among the switching thresholds, the overall performance was controlled by a single parameter, which was the threshold value of Mode 1. In a further contribution, Lau et al. [43] studied the optimization of the adaptive switching thresholds in the context of using conventional M-ary QAM constellations [31]. We note, however, that the design objectives of the M-ary orthogonal modulation and M-ary QAM schemes are rather different, since the former exhibits a higher robustness against transmission errors by sacrificing throughput while the latter

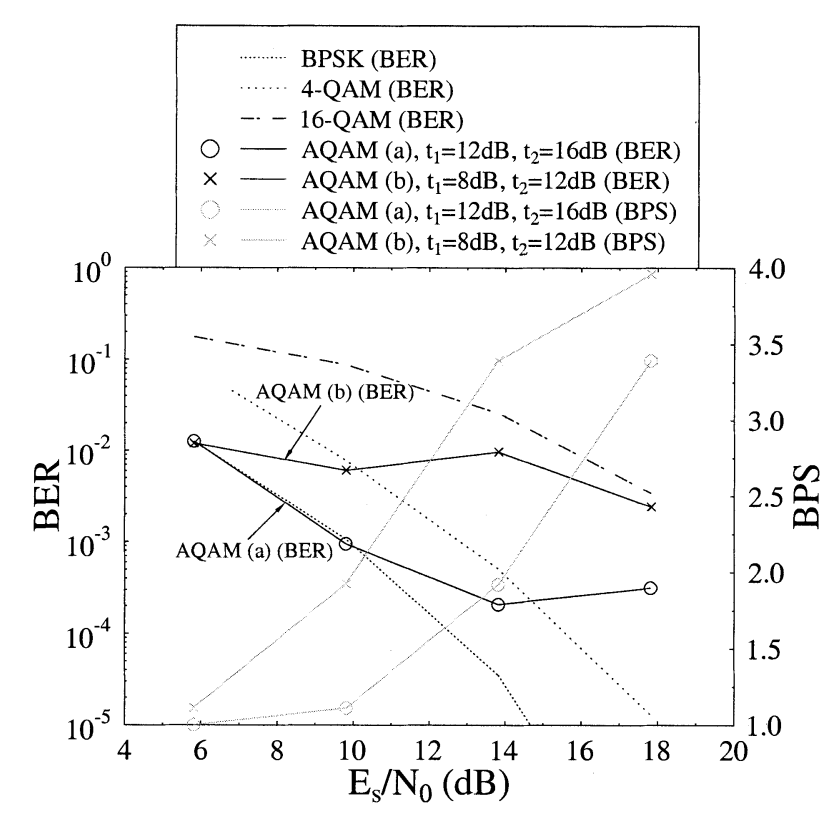

Fig. 3. The average BER and BPS performance versus average $E_{s} / N_{0}$ of an AQAM/JD-CDMA system for $K=2$ users. Three modulation modes were used, namely, BPSK, 4-QAM, and 16-QAM. Two different sets of thresholds were evaluated, as shown in the legends. The BER curves for the same system but using fixed modulation modes of BPSK, 4-QAM, and 16-QAM for $K=2$ users are also shown. The simulation parameters are listed in Table I, and the simulations were conducted over the seven-path Bad Urban channel model of Fig. 2.

achieves a higher throughput at the cost of a reduced error resilience. The associated channel capacities of these schemes have been explicitly characterized in [44, p. 284].

Upon returning to our simulation results, the target BER was set at $1 \%$, which is characteristic of a typical speech transmission system. Since we are employing channel-quality dependent modulation modes for each transmission burst, the performance results are plotted against the average channel SNR perceived by the receiver, namely, against $E_{s} / N_{0}$ on the horizontal axis, where $E_{s}$ represents the energy per QAM symbol. The value of $E_{s} / N_{0}$ can be converted to the corresponding $E_{b} / N_{0}$ value by dividing $E_{s} / N_{0}$ with the number of bits in the QAM symbol. The performance of the AQAM schemes was evaluated by analyzing the BER and the throughput expressed in terms of the average number of BPS transmitted. By evaluating the BER versus $E_{s} / N_{0}$ curves for the three different modulation modes, the required $E_{s} / N_{0}$ values for a BER of $1 \%$ was approximately 10 and $16 \mathrm{~dB}$ for 4-QAM and 16-QAM, respectively. Different sets of thresholds were involved, where the performance obtained using conservative thresholds, i.e., higher thresholds, was contrasted with that of systems using more "aggressive" thresholds-lower thresholds, where a higher BER and BPS throughput was targeted.

The BER performance and BPS throughput comparisons for $K=2$ CDMA users are plotted in Fig. 3. The vertical axis on the left indicates the BER scale, while on the right, the throughput is shown in terms of the average number of BPS. The performance results for two sets of switching thresholds are compared. The more conservative set employed the thresholds of $t_{1}=12 \mathrm{~dB}$ and $t_{2}=16 \mathrm{~dB}$, while the more aggressive set 


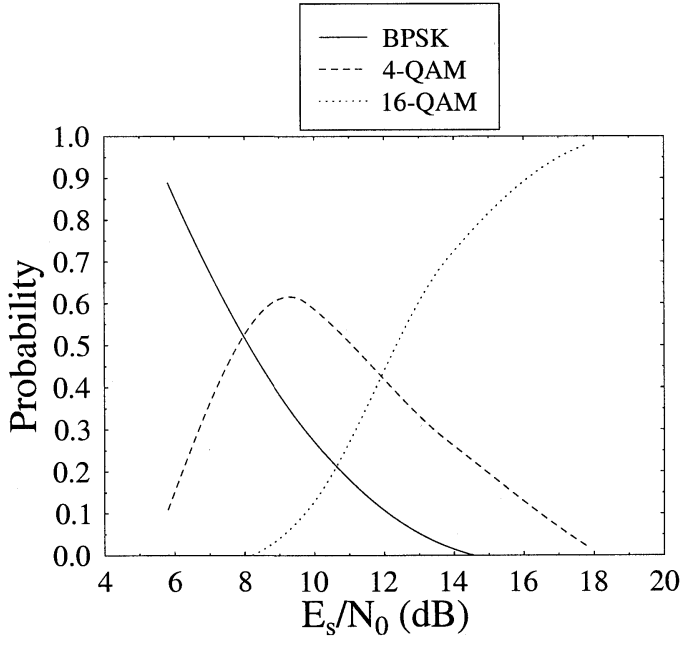

Fig. 4. The probability of each modulation mode's being chosen for transmission in a triple-mode (BPSK, 4-QAM, 16-QAM) two-user AQAM/JD-CDMA system.

used $t_{1}=8 \mathrm{~dB}$ and $t_{2}=12 \mathrm{~dB}$. We also plotted in Fig. 3 the BER performance of fixed-mode BPSK, 4-QAM, and 16-QAM transmissions for reference.

At low values of $E_{s} / N_{0}$, the BPSK mode was chosen with a higher probability, as demonstrated in the modem mode probability distribution function (pdf) of Fig. 4, thus reducing the throughput. However, as $E_{s} / N_{0}$ was increased, the channel quality improved, thus allowing the 4-QAM and 16-QAM modes to be activated more often. This resulted in an increased average BPS performance. Both AQAM systems were capable of maintaining the target BER of $1 \%$ or better. However, the more aggressive thresholds of $t_{1}=8 \mathrm{~dB}$ and $t_{2}=12 \mathrm{~dB}$ gave a higher BPS throughput performance, achieving the maximum of $4 \mathrm{BPS}$ at $E_{s} / N_{0}=18 \mathrm{~dB}$.

The effect of increasing the number of CDMA users in the system was investigated, comparing $K=2$ and 8 . The results are presented in Fig. 5. From the figure, it can be seen that the performance curves for the different number of users match each other quite closely, with only a slight performance degradation, when $K$ was increased from two to eight. This is because the MMSE-BDFE was capable of reducing a significant amount of the MAI by utilizing all the available information regarding the spreading sequences and channel estimates.

In our previous simulations, perfect SINR estimation was assumed. Therefore, an investigation on the effect of imperfect SINR estimation on the AQAM system was conducted, and the results are presented next. Instead of assuming perfect SINR estimation, the output SINR was calculated at the joint detection receiver using the spread sequences and the channel estimates of the received burst. This SINR estimate was then passed on to the transmitter and used as the switching criterion for the next frame to be transmitted. The accuracy of the SINR estimate was therefore dependent upon the speed at which the mobile channel varied. If the channel variations were slow, then the imperfect SINR estimation had a small impact on the chosen modulation mode. Conversely, if the channel variations were fast, then the SINR calculations were less reliable, inflicting a performance degradation. Fig. 6 shows the effects of imperfect SINR estima-

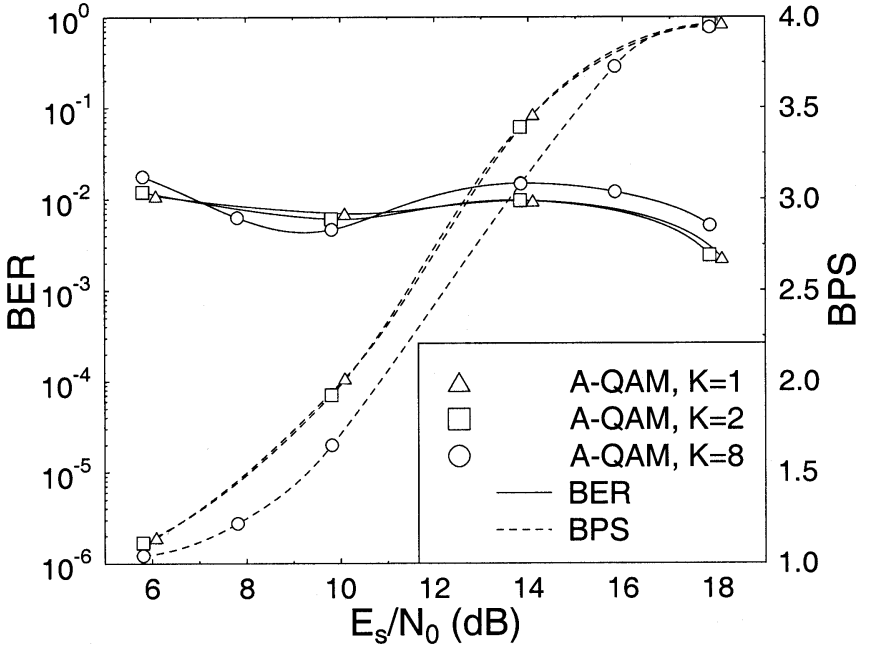

Fig. 5. BER and throughput (BPS) performance comparisons for $K=1,2$, and 8 users in a triple-mode AQAM/JD-CDMA system. The modulation modes used for transmission were BPSK, 4-QAM, or 16-QAM. The simulation parameters are listed in Table I, and the simulations were conducted over the seven-path Bad Urban channel model of Fig. 2.

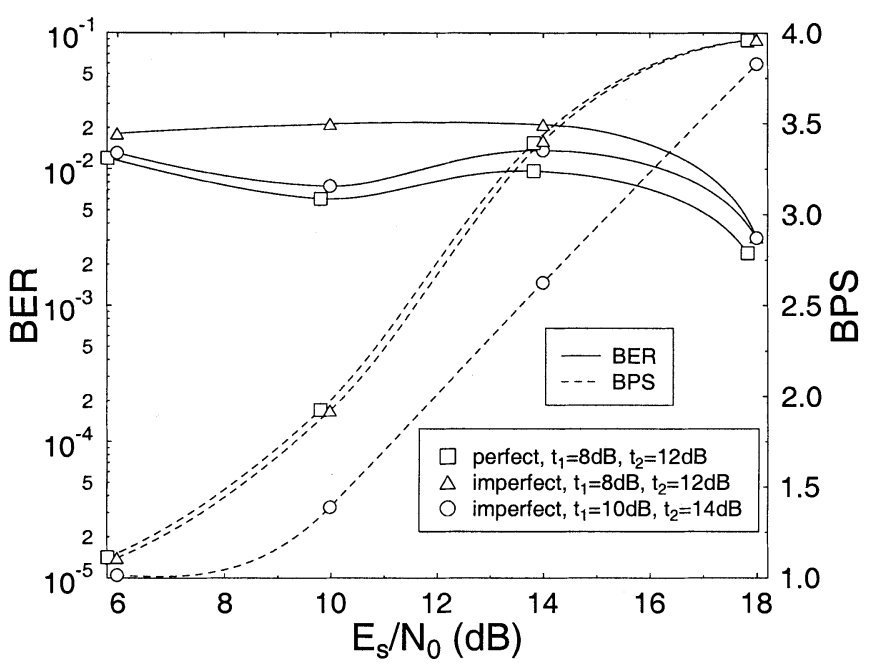

Fig. 6. BER and BPS throughput performance comparisons for a triple-mode AQAM system assisted by perfect and imperfect SINR estimation over the seven-path Bad Urban channel of Fig. 2 at a Doppler frequency of $80 \mathrm{~Hz}$. The symbols $t_{1}$ and $t_{2}$ represent the switching thresholds from BPSK to 4-QAM and from 4-QAM to 16-QAM, respectively. The rest of the simulation parameters are listed in Table I.

tion on the triple-mode AQAM-CDMA system. In our simulations, the TDMA frame structure of FRAMES Mode 1 was used [22], where there were eight bursts per TDMA frame. A worst case scenario was assumed, where a user transmitted one burst per TDMA frame, which led to an eight-burst latency in SINR estimation. The rest of the simulation parameters are listed in Table I.

The imperfect SINR estimation results were compared with the perfect-estimation system that used the switching thresholds of $t_{1}=8 \mathrm{~dB}$ and $t_{2}=12 \mathrm{~dB}$. Two sets of thresholds were tested for the imperfect-estimation system. The first set was the same as that of the perfect-estimation system, but the second set was a higher set of thresholds at $t_{1}=10 \mathrm{~dB}$ and $t_{2}=14 \mathrm{~dB}$. In the first comparison, where both perfect and imperfect systems used 
the same thresholds of $t_{1}=8 \mathrm{~dB}$ and $t_{2}=12 \mathrm{~dB}$, we can see that the inaccuracy in SINR estimation inflicted a BER degradation, since often an inappropriate modulation mode was chosen. When higher threshold values were set, this naturally improved the BER performance at the expense of BPS throughput, as can be seen in Fig. 6 for $t_{1}=10 \mathrm{~dB}$ and $t_{2}=14 \mathrm{~dB}$.

From the results presented here, although imperfect SINR estimation caused BER and BPS degradations, the AQAM method was still capable of delivering adequate BER and throughput performances. Another method that can be used to improve the accuracy of the SINR estimation is the employment of channel prediction techniques. Recent research into mobile channel modeling and adaptive long-range channel prediction by Eyceoz et al. [45] has shown that the mobile channel can be reliably predicted. The predicted CIR can then be used for SINR estimation.

\section{B. VSF-Based ACDMA}

Multirate transmission systems using spreading sequences having different processing gains have been proposed in theliterature by Adachi et al. [46], Ottosson et al. [7], Ramakrishna et al. [8], Saquib et al. [9], and Johansson et al. [10]. In the FRAMES FMA2 wide-band CDMA proposal for the Universal Mobile Telecommunications System [47], different bit rates are accommodated by supporting VSF [46] and multicode operation. In this section, we discuss the use of VSF codes in adaptive-rate CDMA systems. In our proposed VSF system, the chip rate of the CDMA users is kept constant throughout the transmission, while the bit rate is varied by using spreading codes having different spreading factors over the course of transmission. For example, by keeping the chip rate constant, the number of bits transmitted in the same period of time by using a spreading code of length $Q=16$ is twice the number of bits transmitted by using a spreading code of length $Q=32$.

Each user in the VSF-CDMA system is assigned $M$ number of spreading codes with different lengths $Q$. Similarly to the AQAM/JD-CDMA system proposed in Section II-A, the SINR at the output of the MMSE-BDFE is estimated and used as a criterion for choosing the spreading code to be used for transmission. Generally, when the channel is likely to maintain lowerror-rate transmission, a code with a low spreading factor will be used in order to increase the throughput. Conversely, when the channel is hostile, a code having a high spreading factor will be used to minimize the number of errors encountered.

The SINR estimation for this system is not as straightforward as in our proposed AQAM system. The calculation of the SINR requires the knowledge of the spreading sequence used. Since the spreading sequence is the parameter that is being varied, the situation becomes slightly more complicated. One possible method is to calculate the SINR for all possible combinations of the different spreading codes of the different users in the system. However, this would dramatically increase the complexity since it would depend on the total number of users in the system and the number of codes assigned to each user. To simplify the SINR calculations, the spreading sequence having the highest spreading factor (SF) for each user was used in the SINR calculations.

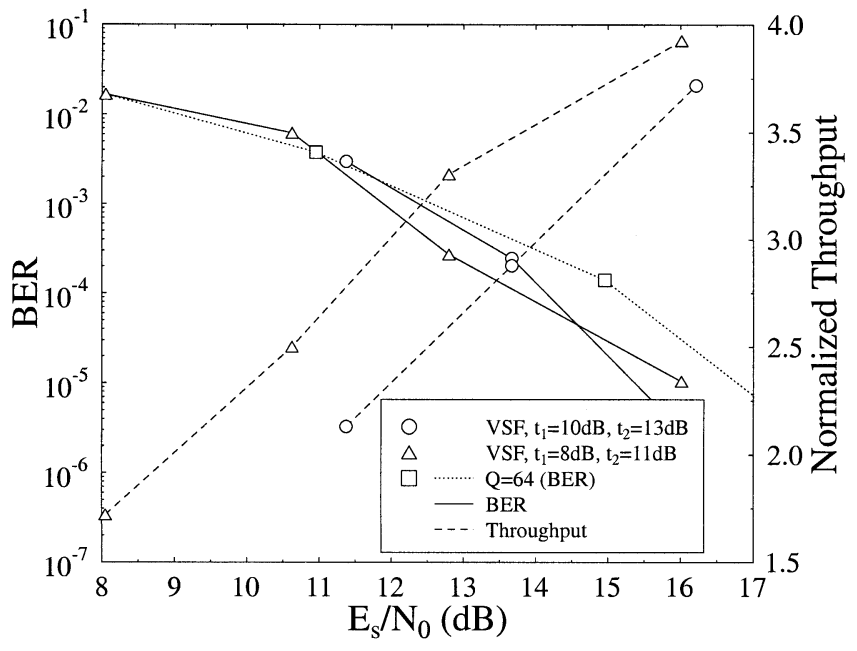

Fig. 7. The average BER and throughput performance versus average $E_{s} / N_{0}$ of a VSF/JD-CDMA system for $K=2$ users over the Bad Urban seven-path channel of Fig. 2. The rest of the simulation parameters are listed in Table I, and perfect SINR estimation was assumed. The transmission rate was varied adaptively by switching among codes having different spreading factors of $Q_{1}=64, Q_{2}=32$, and $Q_{3}=16$. The switching thresholds used are shown in the legends. The throughput values were normalized to the minimum throughput of the system, i.e., the throughput of the fixed-rate $Q=64$-chip system.

In order to have a system that can accommodate a large number of spreading codes having different lengths, the simplest choice of spreading codes would be those with lengths of $Q=2^{r}$, where $r=1,2,3, \ldots$ Let us designate the set of spreading codes assigned to the $k$ th user to be $\left\{\mathbf{c}_{1}^{(k)}, \mathbf{c}_{2}^{(k)}, \ldots, \mathbf{c}_{M}^{(k)}\right\}$, where $\mathbf{c}_{1}^{(k)}$ is the spreading code with the highest $\mathrm{SF}$ and the code $\mathbf{c}_{M}^{(k)}$ has the lowest SF. The rules used to choose the spreading code are as follows:

$$
\begin{aligned}
\gamma_{0}(k)<t_{1} & \Rightarrow \mathbf{c}_{1}^{(k)} \\
t_{1} \leqslant \gamma_{0}(k)<t_{2} & \Rightarrow \mathbf{c}_{2}^{(k)} \\
& \vdots \\
t_{M} \leqslant \gamma_{0}(k) \Rightarrow & \mathbf{c}_{M}^{(k)}
\end{aligned}
$$

where $\gamma_{o}(k)$ is the SINR of the $k$ th user at the output of the multiuser receiver, the values of $t_{1}, \ldots, t_{M}$ represent the switching thresholds for the the modulation modes, and $t_{1}<t_{2}<\cdots<$ $t_{M}$. In our VSF systems, each user maintained a constant level of transmission power for a certain channel SNR (CSNR). This is to ensure that the interference experienced by other users remained relatively similar after compensation for the channel effects at the receiver. The calculation of $E_{s} / N_{0}$ from the CSNR ensued as $E_{s} / N_{0}=\mathrm{CSNR} \times T / T_{c}=\mathrm{CSNR} \times Q$, where $Q=T / T_{c}, T$ is the bit period, and $T_{c}$ is the chip period. Due to the varying value of $Q$, the value of $E_{s} / N_{0}$ will also vary over the course of transmission.

The BER and throughput performance of a triple-mode VSF/JD-CDMA system for $K=2$ users was plotted in Fig. 7, where 4-QAM was used as the data modulation mode. The transmission rate was varied adaptively by switching between the spreading factors of $Q_{1}=64, Q_{2}=32$, and $Q_{3}=16$. Two different sets of threshold values were compared with 


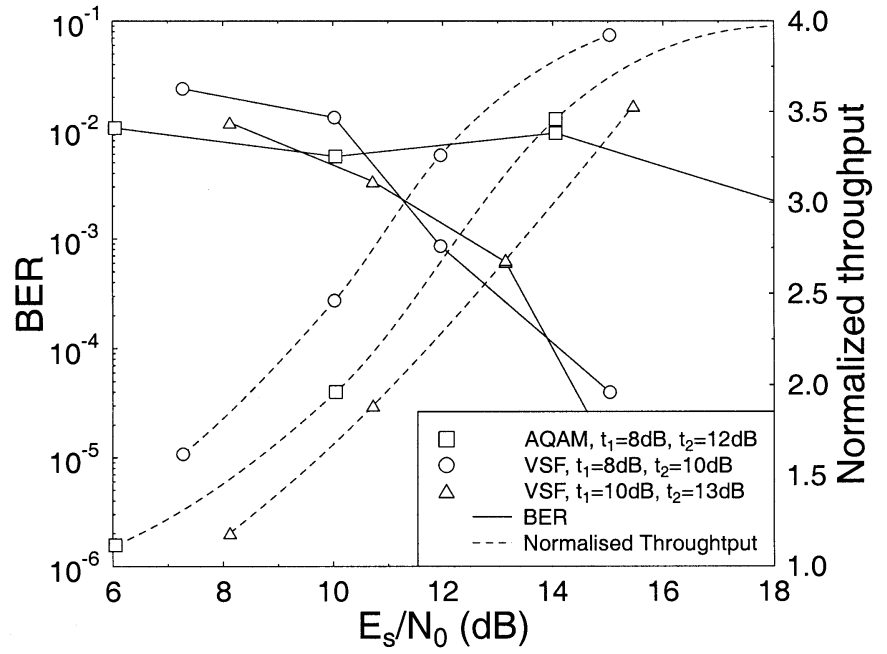

Fig. 8. Comparison of the BER and throughput performance of triple-mode VSF and triple-mode AQAM/JD-CDMA for $K=2$ users. In the AQAM system, the spreading sequences were of $Q=32$ chips in length, and the modulation mode was switched among BPSK, 4-QAM, and 16-QAM. The two VSF systems used 4-QAM and chose the appropriate spreading factor from the set $Q_{1}=64, Q_{2}=32$, and $Q_{3}=16$. The throughput values were normalized to the minimum throughput of the system, i.e., the throughput of the fixed-mode $Q=64$ system. The simulations were conducted over the seven-path Bad Urban channel model of Fig. 2, where perfect SINR estimation was assumed. The rest of the simulation parameters are listed in Table I.

the aim of achieving an average BER of $1 \%$. Specifically, for System $\mathrm{A}, t_{1}=10 \mathrm{~dB}, t_{2}=13 \mathrm{~dB}$; and for System B, $t_{1}=8 \mathrm{~dB}, t_{2}=11 \mathrm{~dB}$ was employed. The resultant throughput performance was normalized to the minimum throughput of the system, giving a normalized throughput ranging from a minimum of one to a maximum of four. The same performance trends were observed in these results as for AQAM. At low $E_{s} / N_{0}$ values, the $Q_{1}=64$-chip spreading code was chosen predominantly, causing the BER to mimic the performance of the fixed-rate $Q_{1}=64$ system. As the transmission power was increased, the throughput performance improved while still maintaining a target BER of $1 \%$. For the lowest threshold values of $t_{1}=8 \mathrm{~dB}$ and $t_{2}=11 \mathrm{~dB}$, a normalized throughput value of approximately 2.3 was achieved for an average $E_{s} / N_{0}$ of approximately $14 \mathrm{~dB}$. For System A with the two higher threshold sets, the BER performance matched that of the fixed-rate $Q=64$ scheme. This was because the adaptive system had the option of choosing a higher spreading factor when the channel was hostile, thus lowering the number of errors in the burst. Let us now analyze the results derived.

\section{Comparison of AQAM and VSF With Joint Detection}

In Section II-A and B, we invoked two different methods of adaptively varying the bit rate of the system, namely, AQAM and VSF. Fig. 8 presents a performance comparison between the two systems. To make a fair comparison, the combination of spreading factors and modulation modes was chosen such that the minimum and maximum throughput of both systems were the same. Specifically, the chosen $K=2$-user AQAM system was a triple-mode scheme that switched modulation modes among BPSK, 4-QAM, and 16-QAM; the spreading factor was $Q=32$. The VSF systems used a modulation mode of 4-QAM and had a choice of three spreading factors: $Q_{1}=64, Q_{2}=32$, and $Q_{3}=16$. The resultant throughput performance was normalized to the minimum throughput of the system, giving a normalized throughput ranging from a minimum of one to a maximum of four. The switching thresholds of $t_{1}=8 \mathrm{~dB}$ and $t_{2}=12 \mathrm{~dB}$ for the AQAM scheme were chosen to obtain a target BER performance of $1 \%$. For the VSF systems, two different sets of thresholds were compared: amore conservative threshold set of $t_{1}=10 \mathrm{~dB}$, $t_{2}=13 \mathrm{~dB}$ and a more aggressive threshold set of $t_{1}=8 \mathrm{~dB}$, $t_{2}=11 \mathrm{~dB}$. From the results presented in Fig. 8, we can see that the AQAM system maintained a fairly constant BER of $1 \%$, while the VSF systems showed a gradual drop in BER as the average $E_{s} / N_{0}$ increased. The constant BER performance of the AQAM system was due to the use of 16-QAM, which allowed a high throughput but exhibited a slightly inferior BER performance at low $E_{s} / N_{0}$ values. Furthermore, at these low $E_{s} / N_{0}$ values, the aggressive VSF system had a slightly worse BER performance than the target of $1 \%$, while the more conservative system was capable of matching the target BER. When comparing the throughput performances, the more aggressive VSF system resulted in a higher throughput than the AQAM system at low $E_{s} / N_{0}$ values, but the throughput was eventually matched by the AQAM system as $E_{s} / N_{0}$ was increased. The most conservative system had a lower throughput than both the other systems.

In comparing the complexities of the three systems, let us take the complexity of the fixed-rate 4-QAM $Q=32$ scheme as a benchmark. The full complexity of the joint detection receiver is on the order of $O(K N)^{3}$ per transmitted burst for the matrix inversion segment of the algorithm, where $K$ represents the number of users and $N$ represents the number of symbols per burst. Various reduced complexity schemes have been proposed that exploit the sparsity of the system matrix [48]-[51]. However, in our subsequent analysis, the full complexity scheme will be utilized. The complexity of the AQAM system was the same as that of the fixed-rate 4-QAM $Q=32$ benchmark scheme because the choice of modulation mode has no effect on the complexity of the JD algorithm or on the setup of the receiver. The VSF schemes had the same complexity as the benchmarker when $Q_{2}=32$ was used, but the complexity decreased eight times when $Q_{1}=64$ was used and increased eight times when $Q_{3}=16$ was used. This was because the use of $Q_{1}=64$ decreased the number of transmitted symbols $N$ by half, while the shorter spreading factor of $Q_{3}=16$ doubled the value of $N$. However, in the two VSF systems, the configuration of the algorithm had to be altered whenever the spreading code of any of the users was changed, thus increasing the complexity.

By contrast, AQAM provides a relatively simple method of adaptively varying the bit rate in order to exploit and accommodate the time-varying nature of the mobile channel without disadvantaging other users. The change in modulation mode does not affect the operation of the joint detection algorithm; thus there is no complexity penalty. However, the use of higher order modulation modes such as 16-QAM increases the BER due to the reduced distance between constellation points. In these cases, the use of VSFs would result in a better BER performance. 


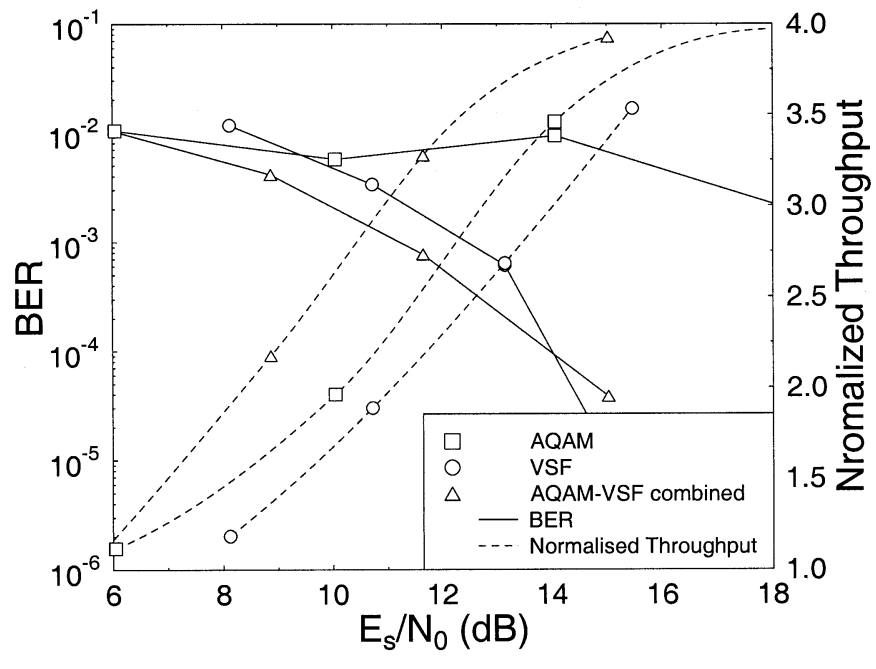

Fig. 9. BER and throughput performance of a $K=2$-user combined AQAM and VSF adaptive-rate JD-CDMA system over the seven-path Bad Urban channel model of Fig. 2. The rest of the simulation parameters are listed in Table I. The bit rate was varied adaptively by using both AQAM and a variable spreading factor. The modulation modes were switched among BPSK with $Q=32$; 4-QAM with $Q=32$; and 4-QAM with $Q=16$. The switching thresholds were set to $t_{1}=8 \mathrm{~dB}$ and $t_{2}=11 \mathrm{~dB}$. Also shown are the performance results for the triple-mode AQAM (BPSK, 4-QAM, 16-QAM) scheme employing $Q=32$, and the triple-mode VSF scheme utilizing 4-QAM as the modulation mode. The throughput values were normalized to the minimum throughput of the system, i.e., the throughput of the mode utilizing BPSK and the spreading factor of $Q=32$.

\section{Combining AQAM With VSF-CDMA}

In order to amalgamate the benefits from both AQAM and VSF-CDMA, a combined AQAM and VSF adaptive-rate JD-CDMA system was simulated and its performance analyzed. Ue et al. [52] investigated a combined symbol-rate and modulation-level controlled adaptive modulation system for TDMA/TDD schemes in narrow-band channels. In our system, the SINR at the output of the MMSE-BDFE was estimated as before, for both the AQAM and the VSF systems. Three different transmission modes were chosen for the combined AQAM VSF-CDMA system: Mode $1-$ BPSK and $Q=32$; Mode 2-4-QAM and $Q=32$; and Mode 3-4-QAM and $Q=16$. The rules used for switching transmission formats were as follows:

$$
\begin{aligned}
\gamma_{0}(k)<t_{1} & \Rightarrow \text { Mode } 1: Q=32 \text { and BPSK } \\
t_{1} \leqslant \gamma_{0}(k)<t_{2} & \Rightarrow \text { Mode } 2: Q=32 \text { and 4-QAM } \\
t_{2} \leqslant \gamma_{0}(k) & \Rightarrow \text { Mode } 3: Q=16 \text { and 4-QAM }
\end{aligned}
$$

where $\gamma_{o}(k)$ is the SINR of the $k$ th user at the output of the MMSE-BDFE. The switching thresholds chosen for maintaining a target BER of $1 \%$ were set at $t_{1}=8 \mathrm{~dB}$ and $t_{2}=11 \mathrm{~dB}$. The results of both simulations are presented in Fig. 9. Also shown for comparison are the triple-mode AQAM scheme using $Q=32$ and the triple-mode VSF scheme using 4-QAM that were characterized in Section II-A-C.

Considering the BER curves, we can see that the combined scheme outperformed both the triple-mode AQAM scheme and the VSF scheme. The target BER of $1 \%$ was achieved, and as the transmission power was increased, the BER also gradually decreased. In throughput terms, the combined scheme also outperformed the other two individual schemes. Having considered the high-complexity JD receivers, let us now focus our attention on a reduced complexity scheme.

\section{INTERFERENCE CANCELLATION}

A different category of multiuser receivers is the class of interference cancellation (IC) receivers that attempt to remove the MAI by reconstructing the original transmitted signals of one or more users and cancelling the interference imposed by these reconstructed signals on the composite received signal [53]. The resultant signal is then processed using the same procedure in order to obtain the data estimates for the remaining users and cancel their interference effects in the received multiuser signal until all the user signals are detected. Generally, the composite received multiuser signal is processed through a first stage that consists of either a bank of matched filters or RAKE receivers. After this stage, initial estimates of the information symbols are obtained and used for reconstruction and cancellation in the subsequent stages. IC techniques are often divided into two categories: successive interference cancellation and parallel interference cancellation [54]. In comparison to the full-complexity JD schemes, IC algorithms have a lower complexity that increases linearly with the number of users. However, the various reduced complexity techniques proposed in the literature have been shown to greatly decrease the complexity of the JD schemes [48]-[51].

SIC [53] receivers rank the users according to received signal quality. Then, the signal of the user with highest signal quality is reconstructed first using the initial data estimates, the CIR, and the spreading sequence of that user. This reconstructed signal is subtracted from the composite received signal. The remaining signal is then processed through the matched filter bank or RAKE receiver of the next strongest signal in order to obtain the data estimates for this user. Employing these data estimates, as well as the CIR and spreading sequence of the user, the transmitted signal is reconstructed and subtracted again from the composite multiuser signal that has already had the strongest user's signal cancelled from it. This is repeated until the data estimates of all the users have been obtained.

In our investigations, the performance of the MMSE-BDFE joint detector is compared to that of the SIC receiver in the context of our burst-by-burst adaptive CDMA system. To generate the initial data estimates for the SIC receiver, RAKE correlators were used for each user, where perfect channel estimation was assumed and maximal ratio combining was employed. The full-complexity MMSE-BDFE has a significantly higher complexity than the SIC receiver. However, the iterative nature of the SIC receiver imposes a substantial delay on the system.

As with the adaptive rate JD-CDMA systems, the criterion used for determining the information rate for the next transmission was chosen to be the SINR at the output of the SIC receiver. The SINR estimate was derived by modifying the approach followed by Patel and Holtzmann [53] for a multipath channel. Having considered a range of uncoded systems, in the 
next section we introduce adaptive trellis coded modulation and turbo TCM, which allow us to mitigate the effects of transmission errors without any bandwith expansion. We will show that further BPS throughput gain can be attained.

\section{AdAPtive Coded Modulation}

A range of adaptive TCM schemes have been investigated in the literature for transmission over narrow-band fading channels [23], [55]-[57]. In particular, adaptive trellis-coded M-ary PSK was considered in [55], and coset codes were applied to adaptive trellis-coded M-ary QAM in [56]. However, these two results were based on some idealized assumptions, such as perfect channel estimation and zero feedback delay. In [57], adaptive TCM using outdated fading estimates was investigated. Recently, the performance of adaptive TCM based on practical considerations such as channel estimation noise, feedback noise, and feedback delay was evaluated in [23]. It was found in [23] that the adaptive TCM scheme is robust in most practical situations.

By contrast, in this section, the performance of the TCM [58] assisted burst-by-burst AQAM JD-CDMA scheme is evaluated when communication is over wide-band fading channels. Imperfect SINR estimation was invoked and the worst case scenario was assumed, where an eight-burst latency was incurred in SINR estimation. The uncoded triple-mode AQAM system's performance assisted by imperfect SINR estimation, as studied in Fig. 6, was compared to that of the TCM assisted AQAM system.

TCM is a powerful forward error correction (FEC) scheme that combines the functions of coding and modulation. It is a bandwidth-efficient scheme that has been widely recognized as an excellent error-control technique suitable for applications in not only fixed Gaussian channels but also mobile communications [59], [60]. The basic idea of TCM is that instead of sending a symbol formed by $\mathrm{m}$ information bits-for example, one information bit for BPSK-we introduce a parity bit while maintaining the same effective throughput of $\mathrm{m}=1 \mathrm{bit} / \mathrm{symbol}$ by doubling the number of constellation points in the original constellation to four, i.e., by extending it to 4-QAM. As a consequence, the redundant bit can be absorbed by the expansion of the signal constellation instead of accepting a $100 \%$ increase in the signalling rate, i.e., bandwidth. A positive coding gain is achieved when the detrimental effect of decreasing the Euclidean distance of the neighboring phasors is outweighted by the coding gain of the convolutional coding incorporated. In conventional uncoded schemes, Gray signal labeling is utilized for increasing the Euclidean distance between the transmitted symbols. By contrast, TCM schemes invoke set partitioning (SP)-based signal labeling in order to achieve a higher Euclidean distance between the unprotected bits of the constellation, so that parallel trellis transitions can be associated with the unprotected data bits. This reduces the decoding complexity. In TCM, a symbol-based random channel interleaver is utilized for dispersing the bursty channel errors.

TTCM [61], is a more recent joint coding and modulation scheme that has a structure similar to that of the family of powerefficient binary turbo codes [62] but employs TCM schemes as component codes. However, TTCM only exhibits superior performance over TCM when the turbo interleaver length used is sufficiently long. There are 56 data symbols in one transmission burst of the TDMA frame structure used [22], which is insufficiently long for achieving a good TTCM performance. The performance of TTCM would be improved using interleaving over several transmission bursts. However, the latency associated with interleaving over several transmission bursts is often unacceptable. Therefore, based on the results of our preliminary experiments using fixed modulation modes, which will be portrayed in Fig. 14, TTCM was not employed in the AQAM system.

In our TCM-assisted burst-by-burst AQAM system, a codeword length of 56 symbols was employed so that there was no increase of latency upon utilizing the TCM scheme, since the received burst was decoded on a burst-by-burst basis. The uncoded triple-mode AQAM system utilized BPSK, 4-QAM, and 16-QAM modulation schemes, providing the throughputs of 1, 2, and 4 bits/symbol. To compare the TCM-based scheme with the uncoded triple-mode AQAM system using the same bandwidth, a TCM-assisted quadruple-mode AQAM system was constructed. The quadruple-mode TCM-assisted system utilized TCM-4-QAM, TCM-8-PSK, TCM-16-QAM, and uncoded-16-QAM while maintaining the throughputs of 1,2 , 3 , and 4 bits/symbol. The coding rate of TCM is $\mathrm{m} /(\mathrm{m}+1)$, where $\mathrm{m}$ denotes the number of information bits per modulated symbol. The generator polynomials of the TCM codes having a memory of three were summarized in Table V. A spreading factor of length $Q=16$ is used, and the rest of the simulation parameters are listed in Table I.

Fig. 14 shows the performance of the TCM- and TTCM-assisted fixed-mode modulation schemes as well as that of the uncoded modulation schemes for $K=2$ CDMA users communicating over the seven-path Bad Urban channel of Fig. 2, which was fading at a Doppler frequency of $80 \mathrm{~Hz}$. Let us compare the coding gain of the TCM schemes against that of the uncoded modulation arrangements having the same bandwidth and throughput at a BER of $10^{-4}$ when communicating over the seven-path Bad Urban COST 207 channel of Fig. 2. For a throughput of $1 \mathrm{bit} / \mathrm{symbol}, \mathrm{TCM}-4-\mathrm{QAM}$ exhibited a 2-dB coding gain against uncoded-BPSK, while for a throughput of 2 bits/symbol, TCM-8-PSK exhibited less than 1-dB coding gain against uncoded-4-QAM. For a turbo interleaver length of 56 symbols, TTCM exhibited no advantage over the less complex memory three constituent TCM codes, as we can observe from Fig. 14.

In light of the above study, TCM schemes were utilized for assisting the burst-by-burst AQAM JD-CDMA system designed for a target BER of $10^{-4}$ using imperfect SINR estimation. Our BER performance and BPS throughput comparisons for $K=4$ CDMA users were plotted in Fig. 15 using the uncoded triple-mode AQAM system and the TCM-assisted quadruple-mode AQAM JD-CDMA system. The BER curves of the AQAM systems followed the trends of their lowest throughput modulation mode and highest throughput modulation mode in the low and high SNR regions, respectively. At medium SNRs, the BER curves of the AQAM systems were maintained at a target BER of $10^{-4}$ by switching between 


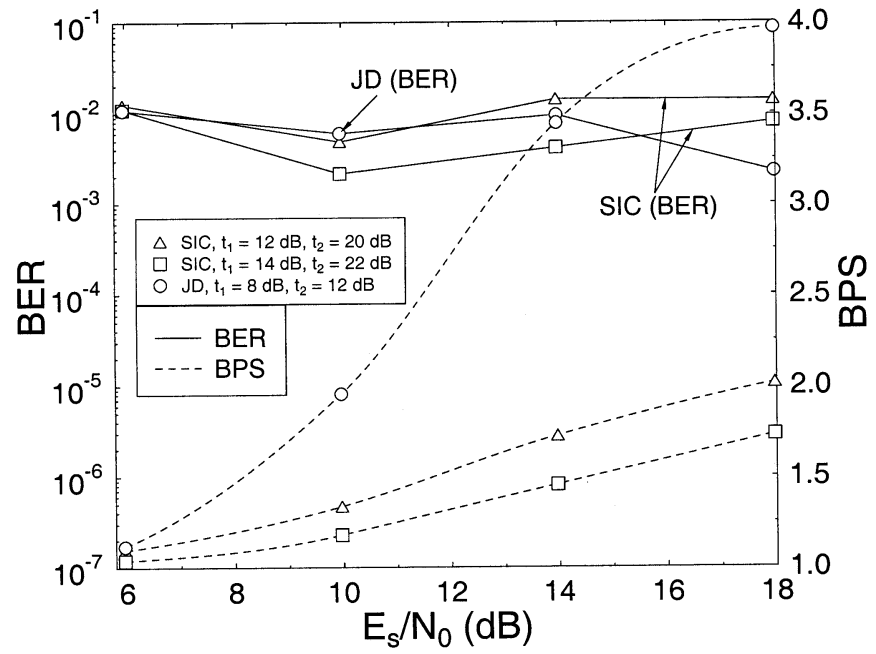

Fig. 10. Comparison of the JD and the SIC receiver for triple-mode (BPSK, 4-QAM, 16-QAM) AQAM transmission and $K=2$ users. A spreading factor of $Q=64$ was used in both systems. The rest of the simulation parameters are listed in Table I. The BER performance is emphasized in (a), while the throughput performance is emphasized in (b).

modulation modes according to the estimated SINR. The performance of the lowest throughput modulation mode and highest throughput modulation mode of the AQAM schemes supporting $K=4$ users was similar to that of the corresponding fixed-mode modulation schemes supporting $K=2$ users, because the MMSE-BDFE is capable of significantly reducing the MAI.

As shown in Fig. 15, the required SNR of TCM-AQAM is $2 \mathrm{~dB}$ lower than that of uncoded-AQAM in the BER region, where we have BER $>10^{-4}$ when supporting $K=4$ users. This trend is similar to that observed in Fig. 14 in the context of fixed-mode TCM-4-QAM and uncoded-BPSK when supporting $K=2$ users. Beyond a channel SNR of $24 \mathrm{~dB}$, the BER curves of both TCM-AQAM and uncoded-AQAM converge to the performance of the uncoded-16-QAM. The BPS performance of the TCM-AQAM scheme is also better than that of the uncoded-AQAM scheme for the channel SNR region spanning from 14 to $24 \mathrm{~dB}$. However, the throughput gains obtained by the TCM scheme are rather limited in this constant bandwidth system despite the higher complexity of the TCM-aided system.

\section{Discussions}

\section{A. AQAM Comparisons}

The performance of the SIC receiver is dependent on the accuracy of the initial data estimates provided by the RAKE receivers. The BER performance of RAKE receivers improves as the spreading factor $Q$ increases. Therefore, in our AQAM comparisons, we will use a spreading factor of $Q=64$ instead of the $Q=16$ that has been used in the AQAM/JD-CDMA systems discussed in earlier sections.

Fig. 10 shows the performance comparisons between the JD receiver and the SIC receiver in the triple-mode AQAM scheme for $K=2$ users, where the modulation mode was switched

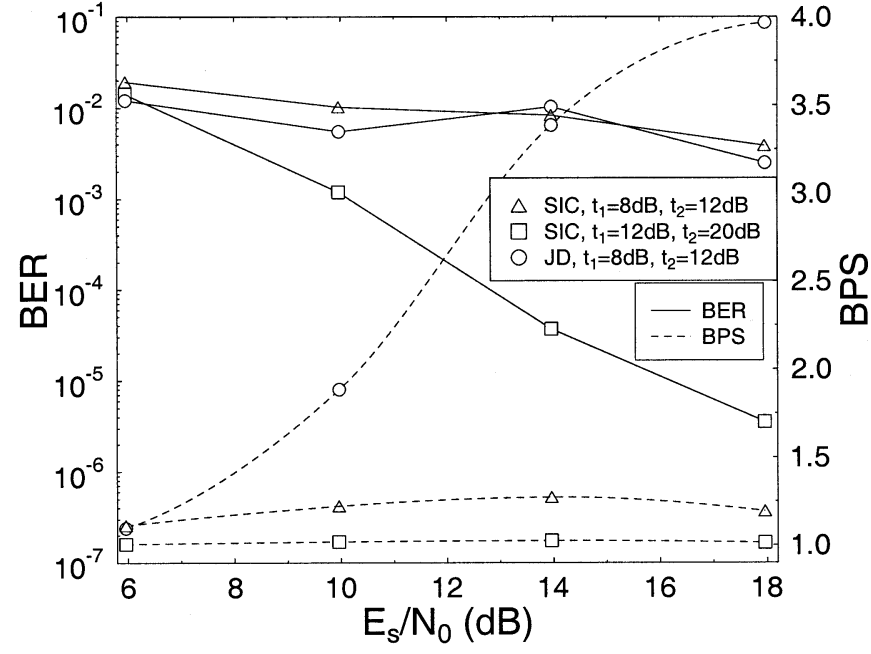

Fig. 11. BER and BPS performance comparisons for triple-mode AQAM schemes with $K=8$ users. The modulation mode was chosen to be BPSK, 4-QAM, or 16-QAM. Two different multiuser receivers were compared: the JD and the SIC receiver. The rest of the simulation parameters are listed in Table I.

among BPSK, 4-QAM, and 16-QAM. The associated BER and BPS performances are portrayed in Fig. 10. Two sets of thresholds were implemented for the SIC receiver. The first set had higher thresholds of $t_{1}=14 \mathrm{~dB}$ and $t_{2}=22 \mathrm{~dB}$, while the second set attempted to set up a more aggressive system with thresholds of $t_{1}=12 \mathrm{~dB}$ and $t_{2}=20 \mathrm{~dB}$. From the figure, we can see that using the higher threshold set for the SIC receiver gave it a slight edge in BER performance over the JD receiver, although its BPS throughput could not match that of the joint detector. Decreasing the thresholds resulted in an improvement in BPS throughput but also led to a corresponding increase in BER. This implies that a further decrease in threshold values would only further degrade the BER performance of the SIC receiver. Comparing the BPS throughput of the joint detector with that of the more aggressive SIC system, we can see that the joint detector outperformed the SIC receiver. The use of RAKE receivers as initial data estimators for the SIC receiver resulted in inaccurate first-stage estimates with the inclusion of 16-QAM as a modulation mode.

The performance comparisons between the two multiuser receiver types were extended to systems that had a higher load of $K=8$ users, as portrayed in Fig. 11. For these systems, we can observe from the results that the SIC receiver was unable to match the BPS performance of the joint detector when both multiuser receivers achieved the same BER. This was because the increase in the number of users increased the multiple-access interference, thus degrading the ability of the RAKE receivers to provide reliable data estimates for successive cancellation.

\section{B. VSF Comparisons}

In this section, the JD and the SIC receiver will be compared in the context of adaptive VSF schemes. The spreading factor used was varied adaptively, opting for $Q_{1}=64, Q_{2}=32$, or $Q_{3}=16$. Since the BER performance of the SIC receiver degrades as the spreading factor decreases, the value of $E_{s} / N_{0}$ was not varied when the spreading factor was switched. Therefore, the transmission power of the the signal varied adaptively 


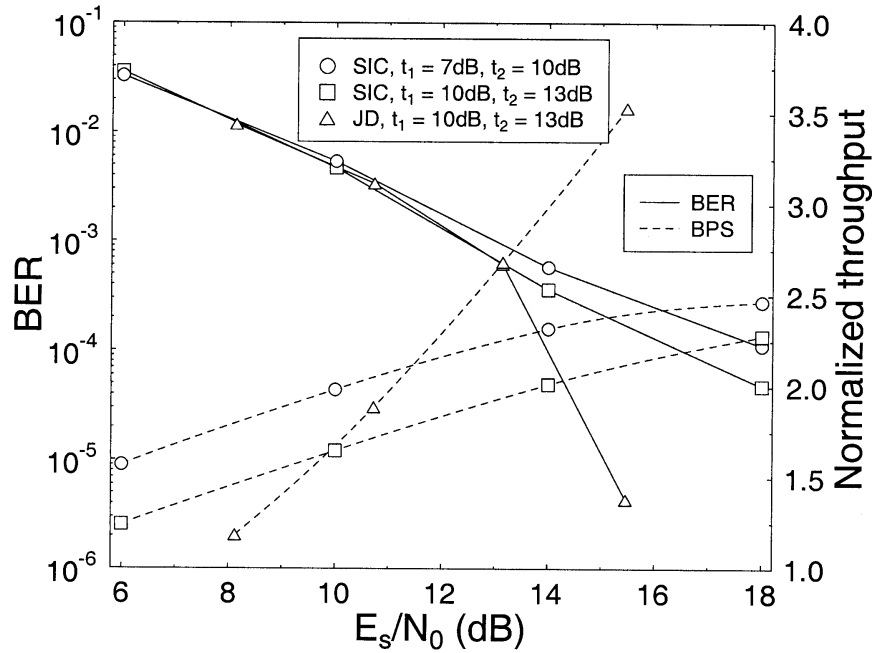

Fig. 12. BER and throughput performance comparisons for triple-mode VSF schemes with $K=2$ users. The spreading factor was adaptively varied using $Q_{1}=64, Q_{2}=32$, or $Q_{3}=16$; and 4-QAM was used as the data modulation mode. Two different multiuser receivers were compared: the JD and the SIC receiver. The rest of the simulation parameters are listed in Table I. The throughput values were normalized to the minimum throughput of the system, i.e., the throughput of the mode utilizing 4-QAM and the spreading factor of $Q=64$.

in order to maintain the constant value of $E_{s} / N_{0}$. This is in contrast to the previous VSF/JD-CDMA schemes, where the transmission power was kept constant in order to maintain a constant interference power to all the other users. Fig. 12 shows the BER and throughput comparisons for the adaptive VSF schemes using 4-QAM. The resultant throughput performance was normalized to the minimum throughput of the system, giving a normalized throughput ranging from a minimum of one to a maximum of four. We compared the JD system to two SIC systems employing different thresholds. The first system had switching thresholds set at $t_{1}=7 \mathrm{~dB}$ and $t_{2}=10 \mathrm{~dB}$, while the second one had higher thresholds of $t_{1}=10 \mathrm{~dB}$ and $t_{2}=13 \mathrm{~dB}$. The switching thresholds set at $t_{1}=10 \mathrm{~dB}$ and $t_{2}=13 \mathrm{~dB}$ for the JD system were chosen for maintaining a target BER of $1 \%$. From Fig. 12 we observe that the SIC system using the lower switching thresholds achieved a higher overall throughput performance at the expense of an inferior BER performance compared to the SIC system invoking the higher thresholds. The employment of higher thresholds allowed the SIC system to achieve a target BER of $1 \%$. The BER and throughput performance of the SIC receiver was inferior in comparison to that of the JD. The JD provided a BER of approximately $0.001 \%$ and a normalized throughput of 2.6 for an average $E_{s} / N_{0}$ of $13 \mathrm{~dB}$ compared to the corresponding throughput value of 2.2 for the same BER and $E_{s} / N_{0}$ values from the more aggressive SIC system.

As seen in Fig. 13, the same comparisons were also performed for the two multiuser receivers based on the VSF scheme with BPSK as the modulation mode. Again, two SIC systems with different thresholds were compared to a JD system with switching thresholds of $t_{1}=6 \mathrm{~dB}$ and $t_{2}=10 \mathrm{~dB}$. The same target BER of $1 \%$ was set. The more conservative set of thresholds for the SIC system was $t_{1}=8 \mathrm{~dB}$ and $t_{2}=$ $11 \mathrm{~dB}$, while the more aggressive set was $t_{1}=6 \mathrm{~dB}$ and $t_{2}=$

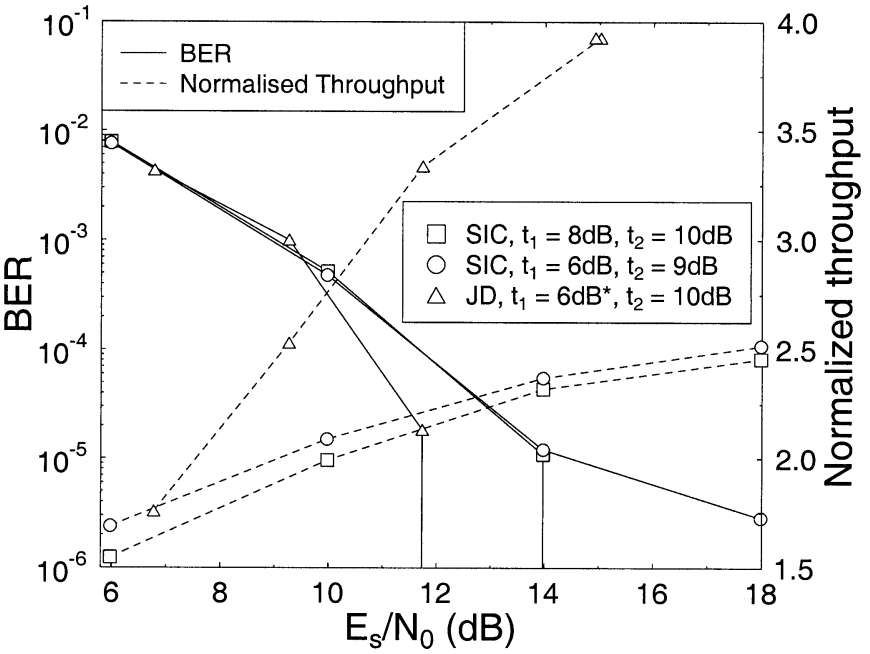

Fig. 13. BER and throughput performance comparisons for triple-mode VSF schemes with $K=2$ users. The spreading factor was adaptively varied using $Q_{1}=64, Q_{2}=32$, or $Q_{3}=16$. BPSK was used as the data modulation mode. Two different multiuser receivers were compared: the JD and the SIC receiver. The rest of the simulation parameters are listed in Table I. The throughput values were normalized to the minimum throughput of the system, i.e., the throughput of the mode utilizing BPSK and the spreading factor of $Q=64$. The BER performance is emphasized in (a), while the throughput performance is emphasized in (b).

$9 \mathrm{~dB}$. Here again, the same performance characteristics were observed as for the previous scheme utilizing BPSK. The JD outperformed both SIC systems, providing a BER of approximately $0.0001 \%$ and a throughput of 3.3 compared to the corresponding throughput value of 2.5 for a similar BER from the more aggressive SIC system.

\section{Conclusions}

Two methods of adaptively varying the transmission bit rate-varying the modulation mode or changing the spreading factor of the spreading code-were proposed. The bit rate was varied in accordance with the quality of the channel. When the channel was of high quality, a higher number of bits was transmitted in order to increase the throughput, while the bit rate was lowered in order to maintain the target BER when the channel was hostile. This allowed us to accommodate and exploit the time-varying nature of the mobile channel. The criterion used for switching the bit rate was the SINR at the output of the multiuser receiver. Our simulation results showed that the adaptive-rate schemes were capable of maintaining a target BER while providing an increased throughput.

The two methods of varying the bit rate were also compared, where the minimum and maximum throughput values available were the same for both methods. It was shown that the VSF method outperformed the AQAM/CDMA system. However, it was an advantage of the AQAM system that the complexity of the JD remained constant despite the change in modulation mode, while the complexity of the VSF receiver varied according to the SF used. Furthermore, the receiver had to be reconfigured for different spreading codes each time the spreading code was changed. It was also demonstrated that a combined AQAM/VSF system, where the bit rate was varied by switching both the modulation mode and the spreading factor, 


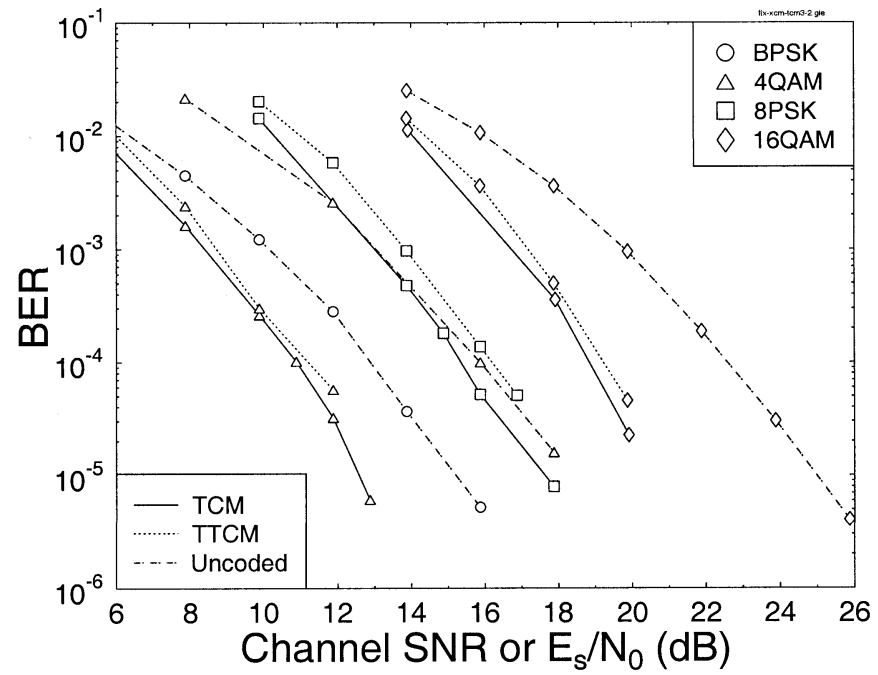

Fig. 14. BER performance comparisons for the fixed modulation modes of the TCM, TTCM, and uncoded schemes when communicating over the seven-path Bad Urban channel of Fig. 2 fading at a Doppler frequency of $80 \mathrm{~Hz}$. Memory 3 TCM schemes and TTCM schemes having memory 3 TCM constituent codes invoking four iterations were compared with the uncoded schemes. The spreading codes were of length $Q=16$, and the number of users was $K=2$. The rest of the simulation parameters are listed in Table I.

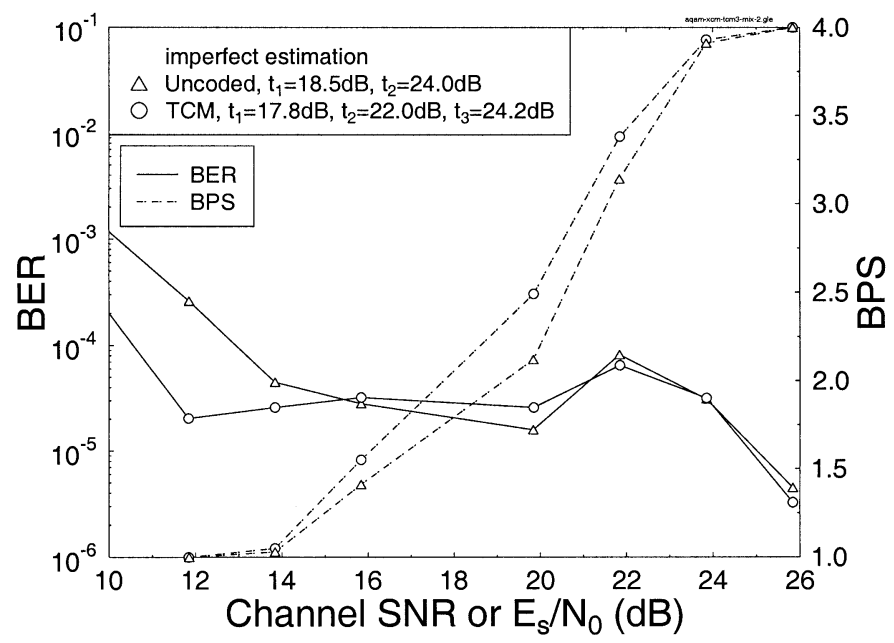

Fig. 15. BER and BPS throughput performance comparisons for the uncoded triple-mode AQAM system and the TCM assisted quadruple-mode AQAM system having imperfect SINR estimation when communicating over the seven-path Bad Urban channel of Fig. 2 fading at a Doppler frequency of $80 \mathrm{~Hz}$. Spreading codes of length $Q=16$ were used, and $K=4$ number of users were supported. The rest of the simulation parameters are listed in Table I.

resulted in improved BER and throughput performances over the purely AQAM or VSF ACDMA systems. Table II summarizes the performance comparisons for the triple-mode AQAM, triple-mode VSF, and triple-mode combined AQAM-VSF ACDMA schemes, where all three schemes are seen to achieve the target BER of $1 \%$ at $E_{s} / N_{0}=8 \mathrm{~dB}$. As the average $E_{s} / N_{0}$ increased, the combined AQAM-VSF ACDMA scheme outperformed the VSF scheme in terms of throughput while still achieving a BER of $0.0001 \%$, which is better than the $0.1 \%$ BER offered by the AQAM scheme.

An adaptive SIC receiver was also investigated, which was outperformed by the JD-based triple-mode AQAM ACDMA
TABLE II

Performance Summary of THE TRIPLE-MOde AQAM, TRIPLE-MOde VSF, AND TRIPLE-MODE AQAM-VSF COMBINED SCHEMES FOR THE JD-CDMA. THE Simulations Were Conducted OVER THE SEVEn-PATH BAD URbaN Channel Model of Fig. 2. The Rest of the Simulation Parameters ARE Listed IN TABLE I. THE NUMBER OF USERS IN THE SySTEM IS

$K=2$. The $E_{s} / N_{0}$ Values ARE The Values at Which EACH System ACHIEVES the BER STATED. THE NOTATION "TP" REPRESENTS THE NORMALIZED THROUGHPUT GIVEN BY THE RATIO OF THE ADAPTIVE SCHEME'S ThroughPUT TO THE THROUGHPUT OF THE FiXED-RATE SCHEME UTILIZING 4-QAM AND $Q=64$, GIVING A Minimum NoRMALIZED ThroughPUT OF ONE AND A MAXIMUM NORMALIZED THROUGHPUT OF FOUR WHEN 4-QAM AND $Q=16$ OR 16-QAM AND $Q=32$ ARE EMPLOYED

\begin{tabular}{c|c|c||c|c|c||c|c|c}
\hline \multicolumn{3}{c|}{ AQAM } & \multicolumn{3}{c|}{ VSF } & \multicolumn{3}{c}{ AQAM-VSF } \\
\hline \hline$E_{s} / N_{0}$ & BER & Tp & $E_{s} / N_{0}$ & BER & Tp & $E_{s} / N_{0}$ & BER & Tp \\
\hline 6 & $1 \%$ & 1.1 & 8 & $1 \%$ & 1.2 & 6 & $1 \%$ & 1.1 \\
18 & $0.1 \%$ & 4 & 15.5 & $0.0001 \%$ & 3.5 & 15 & $0.001 \%$ & 3.9 \\
\hline
\end{tabular}

TABLE III

SUMMARY OF TRIPLE-MODE AQAM-CDMA RESULTS FOR THE JD AND SIC RECEIVERS USING A SPREADING FACTOR OF $Q=64$ AND TRANSMITTING Over the Seven-Path BAD Urban Channel of Fig. 2. The $E_{s} / N_{0}$ Values ARe the Values at Which the AQAM Systems ACHIEVED the TARGET BER OF $1 \%$ OR LESS. THE COMPLEXITY VALUES ARE IN TERMS OF THE NuMBER OF ADDITION PLUS MULTIPLICATION OPERATIONS REQUIRED PER DETECTED DATA SYMBOL

\begin{tabular}{c||c|c|c|c|c|c}
\multicolumn{1}{c||}{$K$} & \multicolumn{3}{c|}{ JD } & \multicolumn{3}{c}{ SIC } \\
\hline \hline & $E_{s} / N_{0}$ & BPS & Cmplx & $E_{s} / N_{0}$ & BPS & Cmplx \\
\hline$K=2$ & 10 & 1.96 & 683 & 10 & 1.34 & 1068 \\
$K=2$ & 14 & 3.44 & 683 & 14 & 1.72 & 1068 \\
\hline$K=8$ & 10 & 1.88 & 2641 & 10 & 1.02 & 1869 \\
$K=8$ & 14 & 3.39 & 2641 & 14 & 1.03 & 1869 \\
\hline
\end{tabular}

TABLE IV

SUMMARY OF TRIPLE-MODE VSF-CDMA RESULTS FOR THE JD AND SIC RECEIVERS, WHERE 4-QAM WAS EMPLOYED AS THE MODULATION MODE AND

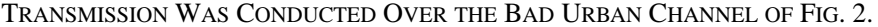

THE NUMBER OF USERS IN THE SYSTEM WAS $K=2$ AND $K=8$. THE $E_{s} / N_{0}$ ARE the Values at Which the VSF Systems ACHIEVED THE TARGET BER OF 1\% OR LESS. THE NOTATION “TP” DENOTES THE ThroughPUt Values Normalized to THE THROUghPUT OF THE FiXed-RATE SCHEME EMPLOYING $Q=64$ AND 4-QAM. THE COMPLEXITY VALUES INDICATED ARE VALID FOR THE MODEM MODE THAT INCURRED THE HIGHEST COMPLEXITY FOR EACH RECEIVER

\begin{tabular}{c||c|c|c|c|c|c}
\hline \multicolumn{1}{c||}{$K$} & \multicolumn{3}{c|}{ JD } & \multicolumn{3}{c}{ SIC } \\
\hline \hline & $E_{s} / N_{0}$ & Tp & Cmplx & $E_{s} / N_{0}$ & Tp & Cmplx \\
\hline$K=2$ & 10.7 & 1.89 & 1496 & 10 & 1.64 & 1068 \\
$K=2$ & 13.1 & 2.68 & 1496 & 14 & 2.01 & 1068 \\
\hline$K=8$ & 11.4 & 2.07 & 18298 & 10 & 1.08 & 1869 \\
$K=8$ & 13.7 & 2.83 & 18298 & 14 & 1.11 & 1869 \\
\hline
\end{tabular}

schemes for a spreading factor of $Q=64$. The SIC receiver exhibited an error floor, especially in its higher order modulation modes. In the VSF systems, the additional use of variable spreading factors of $Q=32$ and $Q=16$ enabled the SIC 
TABLE V

UNGERBÖCK'S TCM CODES, WHERE $\tilde{\mathrm{m}}$ DENOTES THE NUMBER OF INFORMATION BITS TO BE ENCODED OUT OF THE TOTAL OF M INFORMATION BITS IN AN INFORMATION SYMBOL

\begin{tabular}{c|c||c|c|c|c|c}
\hline Code & State & $\tilde{\mathrm{m}}$ & $H^{0}(D)$ & $H^{1}(D)$ & $H^{2}(D)$ & $H^{3}(D)$ \\
\hline \hline 4QAM & 8 & 1 & 13 & 06 & - & - \\
8PSK & 8 & 2 & 11 & 02 & 04 & - \\
16QAM & 8 & 2 & 11 & 02 & 04 & 10 \\
\hline
\end{tabular}

receiver to provide a reasonable BER and throughput performance, which was nonetheless inferior to that of the JD. However, the complexity of the SIC receiver increased linearly with the number of CDMA users $K$ compared to the full-complexity joint detector, which had a complexity proportional to $O\left(K^{3}\right)$. Tables III and IV summarize the performance comparisons for both multiuser detectors in terms of $E_{s} / N_{0}$ required to achieve a target BER of $1 \%$; the throughput performance in terms of BPS or normalized throughput (Tp); and the complexity (Cmplx) in terms of the number of additions and multiplications required per detected symbol.

Finally, the TCM-assisted burst-by-burst AQAM JD-CDMA system was evaluated in conjuction with imperfect SINR estimation. The performance of the TCM-assisted system requiring no bandwidth expansion was compared to that of its uncoded counterpart at a target BER of $10^{-4}$. The TCM-assisted AQAM JD-CDMA scheme exhibited a higher throughput than its uncoded counterpart. However, the throughput gain was modest in this bandwidth-limited scenario. Our future work is focused on joint channel decoding and equalization in the context of so-called per survivor processing assisted JD-ACDMA.

\section{REFERENCES}

[1] K. S. Gilhousen, I. M. Jacobs, R. Padovani, A. J. Viterbi, L. A. Weaver, and C. E. Wheatley, "On the capacity of a cellular CDMA system design," IEEE Trans. Veh. Technol., vol. 40, pp. 303-312, May 1991.

[2] L. Hanzo, C. Wong, and M. Yee, Adaptive Wireless Transceivers. New York: Wiley/IEEE Press, 2002.

[3] J. Blogh and L. Hanzo, $3 G$ Systems and Intelligent Networking. New York: Wiley/IEEE Press, 2002.

[4] L. Hanzo, T. Liew, and B. Yeap, Turbo Coding, Turbo Equalization and Space-Time Coding. New York: Wiley/IEEE Press, 2002.

[5] W. T. Webb and R. Steele, "Variable rate QAM for mobile radio," IEEE Trans. Commun., vol. 43, pp. 2223-2230, July 1995.

[6] S. W. Kim, "Adaptive rate and power DS/CDMA communications in fading channels," IEEE Commun. Lett., vol. 3, pp. 85-87, Apr. 1999.

[7] T. Ottosson and A. Svensson, "On schemes for multirate support in DS-CDMA systems," Wireless Personal Commun., vol. 6, pp. 265-287, Mar. 1998.

[8] S. Ramakrishna and J. M. Holtzman, "A comparison between single code and multiple code transmission schemes in a CDMA system," in Proc. IEEE VTC'98, 1998, pp. 791-795.

[9] M. Saquib and R. Yates, "Decorrelating detectors for a dual rate synchronous DS/CDMA channel," Wireless Personal Commun., vol. 9, pp. 197-216, May 1999.

[10] A.-L. Johansson and A. Svensson, "Successive interference cancellation schemes in multi-rate DS/CDMA systems," Wireless Inform. Networks, pp. 265-279, 1996

[11] S. Abeta, S. Sampei, and N. Morinaga, "Channel activation with adaptive coding rate and processing gain control for cellular DS/CDMA systems," in Proc. IEEE VTC'96, 1996, pp. 1115-1119.
[12] M. Hashimoto, S. Sampei, and N. Morinaga, "Forward and reverse link capacity enhancement of DS/CDMA cellular system using channel activation and soft power control techniques," in Proc. IEEE PIMRC'97, 1997, pp. 246-250.

[13] T. S. Lee and T. C. Tsai, "A partially adaptive CDMA interference canceller for multipath channels," in IEEE Vehicular Technology Conf., vol. 2, May 2000, pp. 917-921.

[14] S. Kazi and L. Lucke, "A convolutionally-coded adaptive CDMA receiver architecture," in Signals, Systems and Computers: 32nd Asilomar Conf., vol. 2, 1998, pp. 1199-1203.

[15] V. K. N. Lau and S. V. Maric, "Variable rate adaptive modulation for DS-CDMA," IEEE Trans. Commun., vol. 47, pp. 577-589, Apr. 1999.

[16] S. W. Lei and V. K. N. Lau, "Adaptive interleaving for OFDM in TDD system," Proc. Inst. Elect. Eng. Commun., vol. 148, no. 2, pp. 77-80, 2001.

[17] L. Hanzo, P. Cherriman, and J. Streit, Wireless Video Communications: From Second to Third Generation Systems, WLAN's and Beyond. Piscataway, NJ: IEEE Press, 2001.

[18] J. S. Blogh, P. Cherriman, and L. Hanzo, "Adaptive beamforming assisted, power controlled dynamic channel allocation for adaptive modulation," in Proc. IEEE VTC'99 Fall, Amsterdam, the Netherlands, 1999.

[19] K. Miya, O. Kato, K. Homma, T. Kitade, M. Hayashi, and T. Ue, "Wideband CDMA systems in TDD-mode operation for IMT-2000," IEICE Trans. Commun., vol. E81-B, pp. 1317-1326, July 1998.

[20] O. Kato, K. Miya, K. Homma, T. Kitade, M. Hayashi, and M. Watanabe, "Experimental performance results of coherent wideband DS-CDMA with TDD scheme," IEICE Trans. Commun., vol. E81-B, pp. 1337-1344, July 1998.

[21] I. Jeong and M. Nakagawa, "A novel transmission diversity system in TDD-CDMA,” IEICE Trans. Commun., vol. E81-B, pp. 1409-1416, July 1998.

[22] T. Ojanperä, A. Klein, and P.-O. Anderson, "FRAMES multiple access for UMTS," in Inst. Elect. Eng. Colloqu. (Dig.), May 1997, pp. 7/1-7/8.

[23] V. K. N. Lau and M. D. Macleod, "Variable-rate adaptive trellis coded QAM for flat-fading channels," IEEE Trans. Commun., vol. 49, pp. 1550-1560, Sept. 2001.

[24] S. Otsuki, S. Sampei, and N. Morinaga, "Square QAM adaptive modulation/TDMA/TDD systems using modulation level estimation with walsh function," Electron. Lett., vol. 31, pp. 169-171, Feb. 1995.

[25] J. Torrance and L. Hanzo, "Demodulation level selection in adaptive modulation," Electron. Lett., vol. 32, pp. 1751-1752, Sept. 1996.

[26] A. Klein and P. W. Baier, "Linear unbiased data estimation in mobile radio sytems applying CDMA," IEEE J. Select. Areas Commun., vol. 11, pp. 1058-1066, Sept. 1993.

[27] A. Klein, G. K. Kaleh, and P. W. Baier, "Zero forcing and minimum mean square error equalization for multiuser de-tection in code division multiple access channels," IEEE Trans. Veh. Technol., vol. 45, pp. 276-287, May 1996.

[28] J. Blanz, A. Klein, M. Nasshan, and A. Steil, "Performance of a cellular hybrid C/TDMA mobile radio system applying joint detection and coherent receiver antenna diversity," IEEE J. Select. Areas Commun., vol. 12, pp. 568-579, May 1994.

[29] P. Jung and J. Blanz, "Joint detection with coherent receiver antenna diversity in CDMA mobile radio systems," IEEE Trans. Veh. Technol., vol. 44, pp. 76-88, Feb. 1995.

[30] P. Jung, J. Blanz, M. Nasshan, and P. W. Baier, "Simulation of the uplink of the JD-CDMA mobile radio systems with coherent receiver antenna diversity," Wireless Personal Commun., vol. 1, no. 1, pp. 61-89, 1994.

[31] L. Hanzo, W. T. Webb, and T. Keller, Single- and Multi-Carrier Quadrature Amplitude Modulation. New York: IEEE Press/Wiley, 2000.

[32] "Office for Official Publications of the European Communities, Luxembourg, COST 207: Digital Land Mobile Radio Communications, Final Rep.,", 1989.

[33] A. D. Whalen, Detection of Signals in Noise. New York: Academic, 1971.

[34] G. H. Golub and C. Loan, Matrix Computations. New York: North Oxford Academic, 1983.

[35] S. Sampei, S. Komaki, and N. Morinaga, "Adaptive modulation/TDMA scheme for large capacity personal multimedia communications systems," IEICE Trans. Commun., vol. E77-B, pp. 1096-1103, Sept. 1994.

[36] A. J. Goldsmith and S. G. Chua, "Variable rate variable power MQAM for fading channels," IEEE Trans. Commun., vol. 45, pp. 1218-1230, Oct. 1997.

[37] J. M. Torrance and L. Hanzo, "On the upper bound performance of adaptive QAM in a slow Rayleigh fading," Electron. Lett., pp. 169-171, April 1996. 
[38] C. H. Wong and L. Hanzo, "Upper-bound of a wideband burst-by-burst adaptive modem," in Proc. IEEE VTC'99, Houston, TX, May 1999, pp. 1851-1855.

[39] J. Torrance and L. Hanzo, "Optimization of switching levels for adaptive modulation in a slow Rayleigh fading channel," Electron. Lett., vol. 32, pp. 1167-1169, June 1996

[40] B. J. Choi and L. Hanzo, "Optimum mode-switching levels for adaptive modulation systems," in IEEE GLOBECOM 2001, Houston, TX, Nov. 2001, pp. 1723-1728.

[41] B. J. Choi, M. Münster, L. L. Yang, and L. Hanzo, "Performance of rake receiver assisted adaptive-modulation based CDMA over frequency selective slow Rayleigh fading channel," Electron. Lett., vol. 37, pp. 247-249, Feb. 2001.

[42] W. H. Press, S. A. Teukolsky, W. T. Vetterling, and B. P. Flannery, Numerical Recipies in C. Cambridge, U.K.: Cambridge Univ. Press, 1992.

[43] V. K. N. Lau, "Performance of variable rate bit interleaved coding for high bandwidth efficiency," in IEEE Vehicular Technology Conf., vol. 3, May 2000, pp. 2054-2058.

[44] J. G. Proakis, Digital Communications, 3rd ed. New York: Mc-Graw Hill, 1995.

[45] T. Eyceoz, A. Duel-Hallen, and H. Hallen, "Determinsitic channel modeling and long range prediction of fast fading mobile radio channels," IEEE Commun. Lett., vol. 2, pp. 254-256, Sept. 1998.

[46] F. Adachi, M. Sawahashi, and K. Okawa, "Tree-structured generation of orthogonal spreading codes with different lengths for forward link of DS-CDMA mobile radio," Electron. Lett., vol. 33, pp. 27-28, Jan. 1997.

[47] A. Toskala, J. P. Castro, E. Dahlman, M. Latva-aho, and T. Ojanperä, "FRAMES FMA2 wideband-CDMA for UMTS," European Trans. Telecommun., vol. 9, pp. 325-335, July-Aug. 1998.

[48] H. R. Karimi and N. W. Anderson, "A novel and efficient solution to block-based joint-detection using approximate Cholesky factorization," in Proc. IEEE Int. Symp. Personal, Indoor and Mobile Radio Communications (PIMRC), Boston, MA, Sept. 8-11, 1998, pp. 1340-1344.

[49] N. Benvenuto and C. Sostrato, "Joint detection with low computational complexity for hybrid TD-CDMA systems," in Proc. IEEE Vehicular Technology Conf. (VTC Fall), Amsterdam, the Netherlands, Sept. 19-22, 1999, pp. 618-622.

[50] J. Mayer, J. Schlee, and T. Weber, "Realtime feasibility of joint detection CDMA," in Proc. 2nd European Personal Mobile Communications Conf. (EPMCC'97), Bonn, Germany, 1997, pp. 245-252.

[51] M. Vollmer, J. Götze, and M. Haardt, "Joint detection using fast fourier transforms in TD-CDMA based mobile radio systems," in Proc. Int. Conf. Telecommunications (ICT'99), Cheju, Korea, June 1999, pp. 405-411.

[52] T. Ue, S. Sampei, and N. Morinaga, "Symbol rate and modulation level controlled adaptive modulation/TDMA/TDD for personal communication systems," in Proc. IEEE VTC'95, pp. 306-310.

[53] P. Patel and J. Holtzman, "Analysis of a simple successive interference cancellation scheme in a DS/CDMA system," IEEE J. Select. Areas Commun., vol. 12, pp. 796-807, June 1994.

[54] M. K. Varanasi and B. Aazhang, "Multistage detection in asynchronous code-division multiple-access communications," IEEE Trans. Commun., vol. 38, pp. 509-519, Apr. 1990.

[55] S. M. Alamouti and S. Kallel, "Adaptive Trellis-coded multiple-phasedshift keying Rayleigh fading channels," IEEE Trans. Commun., vol. 42 pp. 2305-2314, June 1994

[56] A. J. Goldsmith and S. Chua, "Adaptive coded modulation for fading channels," IEEE Trans. Commun., vol. 46, pp. 595-602, May 1998.
[57] D. Goeckel, "Adaptive coding for fading channels using outdated fading estimates," IEEE Trans. Commun., vol. 47, pp. 844-855, June 1999.

[58] G. Ungerböck, "Channel coding with multilevel/phase signals," IEEE Trans. Inform. Theory, vol. IT-28, pp. 55-67, Jan. 1982.

[59] D. Divsalar and M. K. Simon, "The design of Trellis coded MPSK for fading channel: Performance criteria," IEEE Trans. Commun., vol. 36, pp. 1004-1012, Sept. 1988

[60] , "The design of Trellis coded MPSK for fading channel: Set partitioning for optimum code design," IEEE Trans. Commun., vol. 36, pp. 1013-1021, Sept. 1988

[61] P. Robertson and T. Wörz, "Bandwidth-efficient turbo Trellis-coded modulation using punctured component codes," IEEE J. Select. Areas Commun., vol. 16, pp. 206-218, Feb. 1998

[62] C. Berrou, A. Glavieux, and P. Thitimajshima, "Near shannon limit error-correcting coding and decoding: Turbo codes," in Proc. Int. Conf. Commun., Geneva, Switzerland, May 1993, pp. 1064-1070.

Ee-Lin Kuan received the B.Eng degree in electronics engineering (with firstclass honors) and the Ph.D. degree in mobile communications from the University of Southampton, Southampton, U.K.

Her current research interests are associated with mobile transceiver designs, with emphasis on adaptive CDMA techniques.

Soon Xin Ng received the B.Eng degree in electronics from the University of Southampton, Southampton, U.K., in 1999, where he is currently pursuing the Ph.D. degree in the Department of Electronics and Computer Science.

His research interests are mainly in adaptive coded modulation, channel coding, turbo coding, and space-time coding.

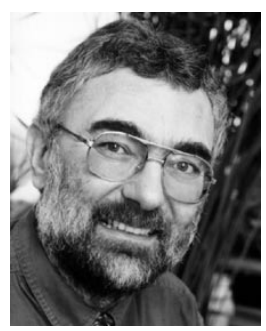

Lajos Hanzo (M'91-SM'92) received the degree in electronics in 1976 and the doctorate degree in 1983, both from the Technical University of Budapest, Budapest, Hungary.

During his 26-year career in telecommunications, he has held various research and academic posts in Hungary, Germany, and the U.K. Since 1986, he has been with the Department of Electronics and Computer Science, University of Southampton, Southampton, U.K., where he holds the Chair in Telecommunications. He has coauthored eight books on mobile radio communications, published about 400 research papers, organized and chaired conference sessions, presented overview lectures, and been awarded a number of distinctions. Currently, he is managing an academic research team working on a range of research projects in the field of wireless multimedia communications sponsored by industry, the Engineering and Physical Sciences Research Council (EPSRC) U.K., the European IST Programme, and the Mobile Virtual Centre of Excellence, U.K. He is an enthusiastic supporter of industrial and academic liaison and offers a range of industrial courses.

Prof. Hanzo is an IEEE Distinguished Lecturer. 